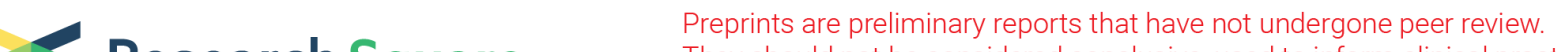 $\begin{array}{ll}\text { Research Square } & \text { They should not be considered conclusive, used to inform clinical practice, } \\ \text { or referenced by the media as validated information. }\end{array}$
}

\section{Proteomic Comparison of Aged and OPTN E50K Retina in the Development of Normal Tension Glaucoma}

\section{Xinna Liu}

The Second Affiliated Hospital of Harbin Medical University

\section{Qi Wang}

The Second Affiliated Hospital of Harbin Medical University

\section{Zhengbo Shao}

The second affiliated hospital of harbin medical university

\section{Shiqi Zhang}

The second affiliated hospital of harbin medical university

\section{Mingying Hou}

The second affiliated hospital of harbin medical university

\section{Menglu Jiang}

The second affiliated hospital of harbin medical university

\section{Mengxian Du}

The second affiliated hospital of harbin medical university

\section{Jing Li}

The second affiliated hospital of harbin medical university

Huiping Yuan ( $\nabla$ yuanhp2013@126.com )

The second affiliated hospital of harbin medical university https://orcid.org/0000-0001-8750-6191

\section{Research article}

Keywords: OPTN E50K mutation, normal tension glaucoma, aging, proteomics, mitochondria metabolism

Posted Date: January 11th, 2021

DOl: https://doi.org/10.21203/rs.3.rs-141353/v1

License: (c) (1) This work is licensed under a Creative Commons Attribution 4.0 International License. Read Full License 


\section{Abstract}

Background: Progressive degeneration of retinal ganglion cells (RGCs) is a major characteristic of glaucoma, whose underlying mechanisms are still largely unknown. An E50K mutation in the Optineurin (OPTN) gene is a leading cause of normal tension glaucoma (NTG), directly affecting RGCs without high intraocular pressure and causing severe glaucomatous symptoms in clinical settings. A systematic analysis of the NTG mouse model is crucial for better understanding of the underlying pathological mechanisms for glaucoma.

Methods: To elucidate proteomic and biochemical pathway alterations during NTG development, we established an OPTN E50K mutant mouse model through CRISPR/Cas9. Retinal proteins from resulting mice exhibiting glaucomatous phenotypes were subject to tandem mass tag-labelled quantitative proteomics. Retinal proteomes were analyzed through bioinformatics methods to characterize the molecular and functional signatures of NTG.

Results: We identified 6364 quantitative proteins in our proteomic analysis. Bioinformatics analysis revealed that OPTN E50K mice experienced protein synthesis dysregulation, age-dependent energy defects and autophagy-lysosome pathway dysfunction. Certain biological features, including amyloid deposition, RNA splicing, microglia activation and reduction of crystallin production, were similar to Alzheimer's Disease (AD).

Conclusions: Our study is the first to describe proteomic and biochemical pathway alterations in NTG pathogenesis during disease advancement. Several proteomic signatures overlapped with retinal changes found in the AD mice model, suggesting the presence of common mechanisms between agerelated degenerative disorders, as well as prospective new targets for diagnostic and therapeutic strategies.

\section{Background}

Glaucoma is the leading worldwide cause of irreversible vision loss, with increasing prevalence [1]. It is characterized by the progressive degeneration of retinal ganglion cells (RGCs), resulting in visual impairment and eventual blindness. Reducing intraocular pressure (IOP) is the most practiced therapy for glaucoma in the clinic, but it is unable to reverse visual impairment and prevent progression to blindness, as well as being insufficient for normal tension glaucoma (NTG) patients, whose IOP is always within the normal range. This observation suggests that glaucoma may develop from a complex interaction of multiple factors, aside from high IOP levels alone. Thus, it is critical to investigate molecular changes and specific biomarkers for early diagnosis and new treatment targets. The E50K mutation of the optineurin (OPTN) gene is known to cause NTG, along with yielding particularly severe clinical glaucomatous symptoms [2-4]. Several E50K-mutant cell models have been applied [5-7], but in vivo studies are still rare. Therefore, we used CRISPR/Cas9 gene-editing technology to introduce the E50K mutation into mice to fully mimic NTG pathologies in vivo, followed by tandem mass tag (TMT) quantitative proteomics to 
perform systematic analysis of the biochemical process within both young- and old-age retinas. Bioinformatics analysis were used to understand molecular neuropathology and potential hallmarks of NTG.

Increasing numbers of studies have indicated a possible relationship between glaucoma and Alzheimer's Disease (AD) [8], both of which are age-related neurodegenerative diseases with some common biological features. For instance, aggregates of OPTN was also observed within neurofibrillary tangles and dystrophic neurites in $A D$ [9], though the exact mechanism behind their occurrence is still obscure. Thus, we also compared the proteomic results for those two diseases to better understand the mechanism underlying retinal degeneration and provide new insights for neurodegenerative diseases.

\section{Methods}

\subsection{Experimental design}

The aim of this study was to investigate the molecular and functional characteristics of the NTG mouse model with the OPTN E50K mutation. Protein expression were assessed by quantitative proteomics, combined with liquid chromatography-tandem mass spectrometry (LC-MS/MS) and TMT labeling. Seventy-two retinal samples in total were used, comprising biological triplicates of 2 genotypes (OPTN E50K and wild type) at 2 age-points (3- and 18-months old). They were randomly divided into three 4-plex TMT experimental sets and then analyzed via MS (Fig. 1a). Among 7019 identified proteins, 6364 proteins in all retinal samples were considered as satisfactory for subsequent quantitative and bioinformatics analyses (Fig. 1b).

\subsection{Animal models and their visual conditions}

CRISPR-CAS9 genome editing was used to introduce the OPTN E50K mutation into female C57BL/6J mice, establishing a NTG mouse model. Wild type (WT) served as a control. Mice were housed in the special pathogen-free (SPF) animal facility at the Second Affiliated Hospital of Harbin Medical University. For genotype identification, DNA sequencing was performed on experimental mice, where mutant mice were homozygous for the OPTN E50K mutation (Supplemental Fig. S1). Mice with OPTN E50K mutation at 3- and 18-months were designated as EY and EO, respectively, while the age-matched WT control groups were correspondingly defined as WY and WO.

Before mice were sacrificed for the proteomics analysis, several measuring procedures were conducted to verify their visual conditions [10]. Briefly, IOP was monitored using an Icare Tonometer (Finland) (Supplemental Fig. S2a), and visual function was assessed by a light/dark test (Supplemental Fig. S2bc). Hematoxylin \& eosin staining was used to assess retinal morphology (Supplemental Fig. S2d-f). Western blot analysis and immunostaining both confirmed that OPTN protein dramatically decreased in mutant mice, compared to their age-matched WT counterparts (Supplemental Fig. S2g-i). However, no significant IOP change was present among all 4 groups. Both WT and OPTN E50K mice demonstrated 
age-related visual impairment, with the EO mice displaying more severe retinal degeneration similar to clinical NTG.

\subsection{Quantitative proteomic analysis by LC-MS/MS}

Mouse retinal tissues were subjected to associated sample preparation methods for MS-based proteomics, including protein digestion, peptide labeling, fractionation, and MS analysis. Detailed procedures are described in Supplemental Methods. Raw data files were processed in Maxquant search engine (v.1.5.2.8). Data were matched against the reviewed Mus musculus 10090_SP_20191115 (17,032 sequences, Feb 2018) and further filtered to retain proteins with false discovery rate (FDR) $<1 \%$.

\subsection{Bioinformatics analyses}

Gene set enrichment analysis (GSEA), based on quantifiable proteins, was performed to elucidate the integrated rankings and differences of pathways between groups [11]. Values were derived by permuting the proteins sets 1000 times within a core pathway extracted from Gene Ontology (GO), Kyoto Encyclopedia of Genes and Genomes (KEGG), and cell type signatures. Normalized enrichment score (NES) was the primary statistic for examining gene set enrichment results, and its statistical significance was estimated by the nominal P-value. Significantly-enriched pathways were selected at a nominal Pvalue level of 0.05 (NOM P-val) and FDR q-value of 0.25 (FDR q-val).

Differentially expressed proteins (DEPs), selected by meeting the significance criteria on a two-sample ttest $P$-value $(P<0.05)$ and fold change threshold $(\geq 1.20$ for up-regulation or $\leq 0.833$ for downregulation), were used for further analysis between two comparative groups. DAVID Bioinformatics (version 6.8) [12] were used to classify the GO, especially the molecular function (MF) of the proteins. MF was evaluated by Fisher's exact test to obtain P-values, and their values also ranked from low to high. Canonical pathways, diseases and biological functions, as well as upstream regulators and networks were enriched using Ingenuity Pathway Analysis (IPA, QIAGEN) [13]. Canonical pathway analysis allowed identification of function-specific genes through assessing the P-values obtained from Fisher's exact test. Analytical algorithms of IPA were carried out on DEPs to predict putative upstream regulators, such as kinases and transcription regulators, as well as downstream effects on known biological pathways. They also assigned the magnitude of activation states as a Z-score ( $z>2$ indicates activation, while $z<-2$ represents inhibition) for pathways or regulators, based on the quantitative values of involved proteins. Interactions between proteins were illustrated by networks based on Ingenuity Pathway Knowledge Base, with a cut-off score of 20 (Supplemental Fig. S3).

\subsection{Quantitative real-time PCR (qPCR) analysis}

Total RNA was extracted from retinas with Trizol, then converted into cDNA using Transcription First Strand cDNA Synthesis kit (Roche, UK). Real-time qPCR was carried out according to the manufacturer's protocols with LightCycler® 480 SYBR Green I Master (Roche, UK). mRNA expression for genes of interest was normalized with GAPDH using the $2^{\Delta \Delta c t}$ method.

\subsection{Western blot analysis}


Protein samples were diluted in loading buffer and heated at $95^{\circ} \mathrm{C}$ for $10 \mathrm{~min}$. Equal amounts of protein were electrophoresed on $12 \%$ or $15 \%$ SDS-polyacrylamide gels, then transferred onto polyvinylidene fluoride membranes. Membranes were then blocked with $5 \%$ skim milk solution for 1 hour at room temperature, followed by incubation with primary antibodies against OPTN (Proteintech, 1:1000), LC3B (Cell Signalling Technology, 1:1000), and P62 (Proteintech, 1:1000) overnight at $4{ }^{\circ} \mathrm{C}$. After three washes, the membranes were incubated with horseradish peroxidase-conjugated secondary antibody (1:10000, Zhongshan) for 1 hour at room temperature. Protein bands were visualized by enhanced chemiluminescence (ECL, Biosharp), and quantified with ImageJ.

\subsection{Immunofluorescent staining}

Mice were anesthetized and perfused with $4 \%$ paraformaldehyde (PFA). Eye tissues were fixed with $4 \%$ PFA overnight at $4{ }^{\circ} \mathrm{C}$, placed in a $25 \%$ sucrose gradient for dehydration, and then frozen. Frozen eyes were embedded in Optimal Cutting Temperature media and cut into $6 \mu \mathrm{m}$ sections. After washing with PBS, eye tissue sections were blocked in $0.5 \%$ goat serum for 1 hour, then subsequently incubated in primary antibodies overnight at $4{ }^{\circ} \mathrm{C}$. To examine microglia and astrocytes, anti-lba-1 (1:50, Abcam) and anti-GFAP (1:50, Proteintech) antibodies were used, respectively. Tissue sections were incubated with Alexa488 goat anti-rabbit secondary antibody for 1 hour at room temperature, followed by DAPI for 5 minutes to counterstain nuclei. Images were taken using a fluorescence microscope and quantified with Image-Pro software.

\subsection{Statistics}

All values were presented as mean \pm SEM. Statistical analysis was conducted using Prism version 7.0 (GraphPad Software). Data were analyzed by Student's t-test for two-group comparisons, or one-way analysis of variance (ANOVA) followed by Tukey's post-hoc test for comparisons among 3 or more groups. $\mathrm{P}<0.05$ was considered statistically significant.

\section{Results}

\subsection{The role of the aging process in NTG pathogenesis}

Aging is the primary risk factor for neurodegenerative diseases, including glaucoma $[14,15]$. To investigate the role of senescence in NTG development, we performed TMT-labelled LC-MS/MS proteomics analysis on OPTN E50K and WT control mice retinas, at both young and old ages. This analysis identified 7019 proteins, of which 6364 were quantifiable from all the retinal tissues used (Fig. 1b). GSEA was performed based on those quantified proteins from all the experimental sets. Agerelated biological pathways were identified based on whether the protein expression for a prior-defined set of genes was statistically enriched in old retinas or depleted in young controls under GSEA, and filtered by NOM P-val $<0.05$ and FDR $<25 \%$. The number of KEGG and biological process (BP) gene sets enriched in EO and WO groups were illustrated by the Venn diagram plots in Figs. $1 \mathrm{C}$ and 1D, respectively. For KEGG, 7 sets were EO-specific, 25 WO-specific, and 24 was found for both groups, while 11 was EO-specific, 128 
WO-specific, and 31 was present in both categories for BP (Fig. 1c-d). These findings overall suggested similar aging effects being present in both pathological and healthy conditions for aged mice. The distribution pattern of these enriched proteins within EO and WO groups was assessed through volcano plots with log-transformed protein abundance ratios. The results showed that 134 (77-up, 57-down) DEPs $(P<0.05, F C \geq 1.20$ or $\leq 0.833$ ) were found in EO (Fig. 1e), and 853 DEPs (519-up, 334-down) in WO mice (Fig. 1f). Among those DEPs, 84 were found within both EO and WO groups (Fig. 1g).

Molecular functions for these proteins were then analyzed by the DAVID database (Fig. 2a), where the structural constituent of eye lens category had the most significant P-value and largest number of DEPs, mostly composed of crystallin proteins. It is also worth noting that the crystallin family is also involved in binding unfolded proteins as molecular chaperones. The distribution pattern of different crystallin family members within triplicates from each of the 4 groups was assessed through a heat map (Fig. 2b), which suggested that crystallins were dramatically down-regulated with age, especially for the EO group. This downregulation was also supported by decreased mRNA levels for Crybb3, Cryba2, Cryab, Crybb2, and Crybb1 in accordance with the decreased protein abundance (Figs. 2c-g). As it has been documented that decreased crystallin levels can reduce its neuroprotective effects, rendering retinal neurons more vulnerable to cytotoxic injury and cell death [16], it is therefore worthy to note that a significant crystallin reduction also occurs in EY mice. Owing to this crystallin downregulation among both EO and EY groups, we investigated the $7 \mathrm{KEGG}$ and $11 \mathrm{BP}$ pathways found to be EO-specific and ranked them based on their NES values (Supplemental Table 1). The most significantly upregulated pathways oxidative phosphorylation (OXPHOS), peroxisome and regulation of actin cytoskeleton, as found among the KEGG gene sets, while the most significantly downregulated pathways, as determined by BP, were sensory perception, cell cycle DNA replication and regulation of proteasomal ubiquitin dependent protein catabolic process in EO retinas. These results indicated that aside from the aging process, specific responses from NTG mice are present for the development of the pathological phenotype.

\subsection{Comparative analysis of the E50K mutation-dependent effect on the retina}

We performed GSEA for protein enrichment analysis on biological processes in EO and EY mice, compared with their respective WO and WY controls to figure out the E50K mutation-specific effects. Twenty-nine BP gene sets were found to be specifically associated with the E50K mutation, no matter the age of the mice (Fig. 3a), which were mainly divided into 2 categories, comprising mRNA splicing (Fig. 3b) and transmembrane transport (Fig. 3c). With respect to mRNA splicing, the E50K mutation is suggested to specifically affect RNA processing via spliceosome, while for transmembrane transport, organic acid, drug, and anion transport are affected. Strikingly, 8 of those 29 BP gene sets, all associated

with genetic information processing, demonstrated opposing trends between EY and EO groups, possibly indicating the occurrence of cross-talk between the aging process and the E50K mutation (Fig. $3 \mathrm{~d}$ ). This possibility is supported by the finding that much more BP gene sets are affected in EO versus EY groups (155 in EO versus 10 in EY), suggesting a synergistic effect between the E50K mutation and aging. 


\subsection{Young OPTN-E50K mutants have similar protein expression profiles as old WT mice}

To determine whether EY mice have a similar pattern of protein expression as WO mice, we analyzed BP alterations in EY versus WY, as well as WO versus WY retinas. Indeed, 16 processes were found to demonstrate the same expression trend in both EY and WO groups (Supplemental Table 2). Since we also found similar mRNA and protein expression levels between EY and WO retinas for the crystallin family (Fig. 2b-g), we tried to investigate the underlying relationship between these two conditions. EY retina had 228 up-regulated and 442 down-regulated DEPs present (Fig. 4a). Interestingly, 185 proteins were also identified in WO retina (Fig. 4b) and the majority of them (137 proteins) was downregulated under both conditions. Closer examination of these proteins found that they were classified as ribosomal, crystallin family, or histone proteins. The distribution pattern for triplicates belonging to WY, EY, or WO groups was displayed in a heat map (Fig. 4c). To better understand their underlying relationships, IPA analysis were performed based on DEPs between EY and WO retinas, where the top 6 canonical pathways for EY were EIF2 signaling ( $p=2.51 \mathrm{E}-35)$, regulation of elF4 and p70S6K signaling ( $p=6.31 \mathrm{E}-16)$, mammalian target of rapamycin (mTOR) signaling ( $p=3.47 \mathrm{E}-10)$, coronavirus pathogenesis pathway $(p=1.58 \mathrm{E}-12)$, sirtuin signaling pathway $(p=9.33 \mathrm{E}-07)$, and glutathione-mediated detoxification $(p=1.41 \mathrm{E}-06)$. EIF2 signaling $(p=3.16 \mathrm{E}-33)$, regulation of elF4 and p70S6K signaling $(p=3.98 \mathrm{E}-14)$, and mTOR signaling $(p=1.26 \mathrm{E}-12)$ were also among the top 6 canonical pathways for WO retinas, but the other 3 pathways were $\mathrm{G}$ beta gamma signaling $(p=3.16 \mathrm{E}-14)$, cardiac hypertrophy signaling $(p=6.31 \mathrm{E}-12)$, and CXCR4 signaling ( $p=$ 1.58E-11) (Fig. 4d). Notably, both EY and WO had the same top three pathways, which also extended to their associated proteins. For instance, both EY and WO shared 45 ribosomal subunit proteins whose expression all decreased under their associated reduced EIF2 signaling comparisons. Such inhibition was predicted by their z-scores (-6 in EY/WY, -3.042 in WO/WY). These similarities in protein abundance and pathway operation between EY and WO mice suggest that E50K mutation may advance age-related degenerative changes in retina, thereby yielding a cumulative impact on visual impairment in NTG.

\subsection{Canonical pathways and protein networks involved in accelerating NTG pathogenesis in aged E50K mutant mice}

As the E50K mutation causes severe clinical symptoms at advanced ages, we tried to gain a more detailed understanding of NTG pathological changes in the context of aging. As 155 biological processes were found to be specifically enriched in EO versus WO mice (Fig. 3a), we analyzed the presence of significantly modulated proteins between those 2 groups and identified 1564 DEPs (952 down-regulated, 612 up-regulated; Fig. 5a). A hierarchical clustered heat map of the DEPs reflected opposing protein abundance distributions between EO and WO groups among the three triplicates (Fig. 5b). Diseases and biological functions analysis (Fig. 5c), as well as canonical pathway (Fig. 5d) enrichment was analyzed by IPA on DEPs. The bubble chart (Fig. 5c) showed the top diseases and biological functions present in EO mice, where notably, degeneration of retinal cells was found to be the most enriched. The top canonical pathways induced by E50K mutation were mitochondrial dysfunction ( $p=3.98 \mathrm{E}-18)$, OXPHOS 
( $p=1.26 \mathrm{E}-17)$, synaptogenesis signaling pathway ( $p=2.04 \mathrm{E}-09)$, EIF2 Signaling ( $p=6.03 \mathrm{E}-10)$, mTOR signaling ( $p=2.04 \mathrm{E}-09)$, phototransduction pathway $(p=2.19 \mathrm{E}-08)$, phagosome maturation $(p=2.24 \mathrm{E}-$ $07)$, protein ubiquitination pathway ( $p=8.13 \mathrm{E}-05)$, AMP-activated protein kinase (AMPK) signaling ( $p=$ 8.51E-05), and apoptosis signaling ( $p=3.02 \mathrm{E}-04)$ (Fig. $5 \mathrm{~d}$ ). Mitochondria provides energy for many intracellular biochemical processes via OXPHOS and comprises five key enzyme complexes. In our dataset, 49 DEPs were associated with mitochondrial complexes, including 20 subunits of complex I (NADH dehydrogenase), 1 subunit each for complex II (succinate dehydrogenase) and III (cytochrome bc1 complex), 9 subunits of complex IV (cytochrome-c oxidase) and 18 subunits of complex V (ATP synthase), respectively (Fig. 5e-f).

Upstream pathway analysis of IPA is capable to predict the presence of molecules governing the regulation of a specific cluster of DEPs. One such molecule, mTOR, was identified and predicted to be activated in EO mice (Supplemental Fig. S3a), based on its high z-score (3.622), statistically significant Pvalue $(p=4.11 \mathrm{E}-06)$, and mTOR signaling being enriched as one of the top canonical pathways. IPA analysis is also able to classify DEPs into different functional units found within networks. Interestingly, mTOR was integrated with mitochondrial complexes in the same interaction network associated with cellular development (Score $=23$; Supplemental Fig. S3b). As a protein kinase, mTOR regulates important biological processes, including metabolism, protein synthesis and particularly autophagy, where it serves as a central regulator by modulating multiple aspects of this process, such as initiation, processing, and termination. Active mTOR inhibits autophagy induction, while OPTN serves as an autophagy receptor involved in autophagy induction. E50K mutation negatively affects OPTN expression, leading to the autophagy pathway being disrupted, and resulting in injured RGCs. Even though autophagy was not enriched as one of the top canonical pathways, we observed a number of autophagy-associated proteins with decreased expression in both EY and EO retinas comparing with age-matched controls, including Atg3 (0.72-fold) and Atg4b (0.82-fold) (Supplemental Table 3).

To verify mitochondria-related proteomic results and the predicted autophagy inhibition, we performed qPCR to determine the mRNA levels for selected mitochondrial gene candidates whose expression greatly changed in the EO retina, as well as Western blotting to measure expression of the well-known autophagy markers LC3 and P62 in all four groups. The downregulated mRNA levels for Ndufb8, Ndufb6, Ndufa13, Ndufb5, Cox4i2, Cox4i1 and Atp6v0c, along with upregulated levels for Ndufv3, Cox17 and Atp6v1f in the EO group were consistent with the abundances of their corresponding proteins (Fig. 6a-b). Western blot results also showed increased P62 levels, as well as LC3 II/I ratios, under E50K mutation, which was accentuated with increased age (Fig. 6c-f). Increases in those values are hallmarks of E50K-induced autophagy blockage $[7,17]$. Other key autophagy-associated proteins are SNAREs and RABs, both of which are involved in facilitating autophagosome and lysosome fusion. We found that the functions for 22 SNARE-associated proteins were significantly inhibited for lysosomes (NOM P-val $=2.94 \mathrm{E}-03$, FDR q$\mathrm{val}=3.37 \mathrm{E}-02)$ and SNARE interactions in vesicular transport (NOM P-val $=0.00 \mathrm{E}+00, \mathrm{FDR}$ q-val $=7.55 \mathrm{E}-$ 04) under GSEA in EO mice (Supplemental Fig. S4 and Table 4). As for RABs, 27 proteins in the family were found to be downregulated in the EO group (Supplemental Table 5), while 7 proteins were also found to have reduced expression in the EY group, compared to their age-matched WT counterparts. 
Together, these results suggested that autophagy and its associated pathways were significantly disrupted in NTG retinas, even at younger ages.

\subsection{NTG shares pathological phenotypes with Alzheimer's disease}

We performed GSEA analysis to determine the specific pathological pathways in EO mice involved with NTG development. Among these significantly enriched pathways, those associated with metabolism, as well as the neurodegenerative Alzheimer's (AD) and Parkinson's diseases (PD) were upregulated, while most genetic information processing and degradation-associated pathways were downregulated (Fig. 7a). Recent studies have reported amyloid-beta $(A B)$ deposition, the hallmark of $A D$, also occurring in glaucoma retina, as well as evidence supporting an association between $A D$ and glaucoma through sharing common pathological mechanisms, such as mitochondrial dysfunction [18-20], abnormal accumulation of misfolded proteins [21], glial activation, and inflammation [22-24]. In addition, recent proteomic research have investigated dysregulated mRNA splicing and decreased crystallin in both AD and glaucoma retinas [25-27] (Fig. 7b). OPTN has been observed within neurofibrillary tangles and dystrophic neurites in $A D$, implying it may also be a contributory gene there [9]. Our proteomic results confirmed changes in mitochondria and autophagy of OPTN E50K retinas, as well as the spliceosomeassociated gene set being more enriched under GSEA for EO mice, especially for serine/arginine-rich (SR) proteins including Srsf6 (1.40-fold), Srsf4 (1.35-fold) and Sf3a1 (1.32-fold). The upregulation of these proteins in NTG were verified by qPCR results for their corresponding mRNAs (Figs. 7C-D).

Retinal sections were then stained with Iba 1 and GFAP to confirm the NTG microglia-astrocyte-neuron crosstalk, in which both microglia and astrocytes were mostly located in the ganglion cell layer (GCL) (Fig. 8a). The number of both $\mathrm{Iba}^{+}$and $\mathrm{GFAP}^{+}$cells in the GCL increased with age, especially in EO mice (Fig. 8b-c). From this finding, we postulated that the microglia and astrocytes may also play major roles in neuroinflammatory conditions, such as AD, as both diseases demonstrated similar retinal signatures. This postulation was supported by GSEA showing enrichment of proteins associated with both cell types in EO compared to WO mice (Supplemental Fig. S5). We tried to determine whether NTG increases the likelihood of $A D$ susceptibility by examining $A \beta$ deposition through retinal immunostaining, where it was increased in E50K mutant mice compared to their age-matched WT counterparts (Fig. 8d-e). Another common risk factor between NTG and AD was the crystallin family, which was demonstrated under immunostaining for both Crybb3 and Cryab proteins (Fig. 8D, f-g). Lower expression levels were found for the EO group, compared to the WO group, for both proteins, while downregulation was only present for Cryab in EY mice compared to WY ones (Fig. 8f-g) [26, 27]. These data revealed that NTG with OPTN E50K mutation is a widespread age-related neurodegenerative condition, which shares common pathological phenotypes with AD in terms of glial cell activation, reduced crystallin production, and $\beta$ amyloid aggregation.

\section{Discussion}


OPTN E50K mutation is the most common mutation for NTG [2, 3], causing severe symptoms in patients [4]. Its pathological mechanism is still obscure, and systematic analysis is rare. In the present study, CRISPR/Cas9 was used to produce OPTN E50K mice. Proteomic analysis was performed to elucidate proteomic and biochemical pathway alterations during NTG development with respect to mitochondrial function, autophagy, and protein synthesis. Comparative analysis between NTG and AD also provided evidences for shared retinal pathological manifestations, suggesting common pathogenesis between those disorders.

CNS neurons are highly energy-demanding cells, dependent on mitochondrial OXPHOS [19]. Thus, mitochondrial dysfunction is a major cause of aging and neurodegenerative disease, including glaucoma [28]. In old E50K mice, 180 DEPs are identified as mitochondrial matrix and membrane components. Complex I, the largest multi-subunit complex, coupling NADH-ubiquinone electron transfer with the generation of a proton gradient for ATP synthesis, was the most severely affected in old E50K mice [29, 30]. Damage to that complex is associated with ROS generation [29], triggering oxidative stress and gradual cell death, thereby partly explaining the previous finding of E50K mutation selectively inducing ROS-mediated RGC death [31]. Another factor behind this mechanism is complex IV disruptions, of which 16 DEPs were found in old OPTN E50K mice. RGCs, unlike other neurons, are directly exposed to light, thus cytochrome oxidase (COX) in complex IV is important to help absorb light and avoid retinal damage [32], which was lost with complex IV dysfunction. A key molecule in energy homeostasis is the AMPK complex, which senses low cellular ATP levels to trigger autophagy and inhibit protein synthesis in response to energetic stress and mitochondrial insults [33,34]. However, this capacity is attenuated under aging and pathological conditions, where severe mitochondrial damage disturbs AMPK signaling, causing apoptosis [33]. The finding of these associations is in agreement with our IPA results in old OPTN E50K mice indicating significant inhibition in OXPHOS and AMPK signaling.

Autophagy is a fundamental cellular process mediating cellular component degradation, and defects in this process plays an important role in neurodegenerative disorder pathogenesis $[35,36]$. mTOR is its central regulator, found in our IPA analysis to target various downstream effectors, such as PI3K/AKT signaling, mitochondrial protein synthesis, and mitochondrial-lysosomal homeostasis. Recently, mitochondria and lysosomes are considered mutually functional in maintaining homeostasis [37, 38], where mitochondrial dysfunction leads to lysosomal impairment and autophagy by-product buildup, whereas lysosomal defects trigger functional and morphological mitochondrial defects. This is supported by our IPA analysis showing that mTOR signaling-associated canonical pathways of mitochondria function and phagosome maturation were both disrupted. This connection between mTOR, mitochondria, and lysosomes is facilitated by V-ATPase, which ensures low lysosomal pH to maintain lysosomal hydrolase activity and autophagic vesicle fusion [39, 40]. Loss of V-ATPase subunits triggers accumulation of non-functional lysosomes and inhibition of autophagy, as shown in a previous study where downregulation of the Atp6v0c subunit is observed to promote accumulation of autophagosomes with high basal LC3 II levels, thereby adversely affecting autophagy-lysosome pathway (ALP) function [41]. This was consistent with our findings showing Atp6v0c down-regulation, coupled with higher LC3 II levels, indicating autophagy dysfunction, in old OPTN E50K mice. Furthermore, our findings indicating 
SNARE family inhibition and RAB family downregulation are in accordance with reduced autophagy activity being present in E50K mutants, as both protein families are associated with membrane fusion. We thus speculate that E50K mutant autophagy blockage stems from the impairment of multiple cellular pathways.

Our results showed the OPTN E50K mutation inducing several age-related proteomic features, even at younger ages. Similar DEP distribution was found between proteins in young OPTN E50K and old WT retinas, particularly those associated with EIF2, elF4, p70S6K, and mTOR signaling, as well as those found in the ribosome, all of which are involved in protein synthesis and energy metabolism, suggesting a common energy stress response occurring in both retinal types. This association, particularly with ribosomal proteins, is supported by recent studies highlighting that ribosomal dysfunction may cause neuronal synaptic functional and plasticity impairment $[42,43]$. The similarities for DEPs are further supported by our GSEA analysis finding 16 biological processes, associated with transcription, translation and protein folding in endoplasmic reticulum, being affected in both young OPTN E50K and old WT retinas, with the same trends. Interestingly, 8 processes for young OPTN E50K mice were discovered to also be affected with old mutants, but with opposite trends. We postulate that aging-driven dysfunctions in transcriptional and protein homeostasis play an important role in old-age glaucoma, which is aggravated by the E50K mutation. This is consistent with clinical findings showing that NTG patients with E50K mutation had early and severe visual dysfunction.

The retina responds similarly to the brain under pathological conditions, owing to it being an extension of the CNS $[44,45]$. Being the only place allowing direct visualization of neurons and blood vessels, early non-invasive detection and monitoring of cerebral changes can take place, resulting in greater interest in identifying ocular indicators for neurodegenerative diseases, such as $A D$ [44, 46-48]. Various studies have proven $A D$ ocular involvement, including RGC loss, retinal thinning, astrogliosis, inflammatory changes, and $A \beta$ plaques $[47,48]$. Some of these processes, particularly RGC loss, GCL thinning, and $A \beta$ presence, are also present in glaucoma $[49,50]$. A recent study also observed OPTN within AD neurofibrillary tangles and dystrophic neurites [9], supporting our usage of the OPTN E50K mouse model to investigate underlying common pathologies between NTG and AD. We first examined A $\beta$ deposits within NTG retinas, and observed its age-related elevation and disease-specific enhancement in OPTN E50K mice, even at early stages. This aggregation was also observed at 2-3 months in AD mice model retinas, prior to their brain appearance [51]. Based on this observation, it is likely that the OPTN E50K mutation may be connected to $A \beta$ accumulation for both NTG and $A D$ through its impairment of autophagy, which would otherwise remove $A \beta$ peptides [52-54]. Another important pathologic process for both diseases is neuroinflammation triggered by astrocytes and microglia in response to protein aggregates and degenerated neurons $[49,55]$. Its occurrence was supported by our findings where astrocytes and microglia were significantly activated in old OPTN E50K mice. Both AD and NTG share other pathological biochemical alterations, such as mitochondria, mRNA splicing and crystallin protein dysfunction [27, 56]. In particular, alternative splicing dysregulation is a common characteristic for several nervous system age-related illnesses, and glaucoma is no exception, as our GSEA analysis showed that the spliceosome was significantly up-regulated in old OPTN E50K mice, involving 45 DEPs 
[57-59]. As for crystallin proteins, involved in eye development and protein folding, their levels were decreased in both NTG and AD mice [26, 27, 60-63]. Indeed, a reduction in abundance was observed in previous studies for 7 such proteins in association with high IOP [26, 64], as well as here for young OPTN E50K mice. In particular, 2 crystallin proteins within those 7, Crybb3 and Cryab, were also found to have significantly decreased in AD mice retina [27], further supporting findings highlighting biomolecular similarities between glaucoma and AD. We also found this crystallin decrease to be associated with neurofibril formation and glial cell inflammation, consistent with previous studies [61-63]. Overall, the shared pathological alterations between NTG and AD provide promising targets for glaucoma treatment, as well as possible markers to diagnose CNS disease in patients.

\section{Conclusion}

Our study elucidated that the OPTN E50K mutation is associated with aggravating several age-related changes at the early stages of NTG, resulting in young mutants sharing similar phenotypes as old WT mice. These changes, such as with mitochondrial energy metabolism, autophagy, and mTOR signaling, were further accentuated in OPTN E50K retinas, suggesting glaucoma resulting from a complex interaction of multiple cellular processes. We also demonstrated the role of energy defects and ALP impairments via AMPK-mTOR pathway in NTG retinal degeneration, and further outlined the biochemical connections with the neurodegenerative disease $A D$, which may aid in find certain retinal biomarkers for accessible and non-invasive CNS disease detection.

\section{Abbreviations}

RGCs: Retinal ganglion cells

NTG: Normal tension glaucoma

AD: Alzheimer's Disease

IOP: Intraocular pressure

OPTN: Optineurin

TMT: Tandem mass tag

LC-MS/MS: Liquid chromatography-tandem mass spectrometry

WT: Wild type

SPF: Special pathogen-free

GO: Gene Ontology

KEGG: Kyoto Encyclopedia of Genes and Genomes 
NES: Normalized enrichment score

DEPs: Differentially expressed proteins

MF: Molecular function

IPA: Ingenuity Pathway Analysis

OXPHOS: Oxidative phosphorylation

BP: Biological process

mTOR: Mammalian target of rapamycin

AMPK: AMP-activated protein kinase

PD: Parkinson's diseases

$A \beta$ : Amyloid-beta

SNARE: Soluble NSF Attachment Protein Receptor

ALP: Autophagy-lysosome pathway

\section{Declarations}

\section{Ethics approval and consent to participate}

Animals were treated and maintained as per the Helsinki Treaty. All experimental protocols were approved by the Institutional Animal Care and Use Committee at Harbin Medical University and conformed to the Guide for the Care and Use of Laboratory Animals (NIH, 8th Edition, 2011). This study was approved by Ethics Committee of the Second Affiliated Hospital of Harbin Medical University (Permit Number: KY 2018-220).

\section{Consent for publication}

Not applicable.

\section{Availability of data and materials}

Raw data for the current study is available from the corresponding author upon reasonable request.

\section{Competing interests}


The authors declare that there are no conflicts of interest.

\section{Funding}

This work was supported by the National Natural Science Foundation of China (81870654 to H-PY, 82070956 to H-PY, 81970799 to Z-BS); Applied Technology Research and Development Program of Heilongjiang Provincial Science and Technology Department (GA20C008 to H-PY); Heilongjiang Postdoctoral Scientific Research Developmental Fund (LBH-Q18082 to Z-BS).

\section{Authors' Contributions}

X-NL, QW and Z-BS contributed to the design of the study, analysis and interpretation of the data, and wrote the manuscript; S-QZ assisted with immunostaining, behavior test and proteomic analysis; $\mathrm{M}-\mathrm{YH}$ contributed to sample collection and immunostaining; M-LJ, M-XD and JL contributed to the literature search, data acquisition and manuscript preparation; H-PY contributed to conception and design, final approval of manuscript and funding acquisition. All authors read and approved the final manuscript.

\section{Acknowledgements}

We thank Dr. Alina Yao for assistance with manuscript writing and Jingjie PTM BioLab (Hangzhou), Co. Ltd for helpful directions.

\section{References}

1. Weinreb RN, Aung T, Medeiros FA. The pathophysiology and treatment of glaucoma: a review. JAMA. 2014; 311(18): 1901-11.

2. Iwase A, Suzuki Y, Araie M, Yamamoto T, Abe H, Shirato S, et al. The prevalence of primary openangle glaucoma in Japanese: the Tajimi Study. Ophthalmology. 2004; 111(9): 1641-48.

3. Song W, Shan L, Cheng F, Fan P, Zhang L, Qu W, et al. Prevalence of glaucoma in a rural northern china adult population: a population-based survey in kailu county, inner mongolia. Ophthalmology. 2011; 118(10): 1982-88.

4. Minegishi Y, Nakayama M, lejima D, Kawase K, Iwata T. Significance of optineurin mutations in glaucoma and other diseases. Prog Retin Eye Res. 2016; 55: 149-181.

5. Sayyad Z, Sirohi K, Radha V, Swarup G. $661 \mathrm{~W}$ is a retinal ganglion precursor-like cell line in which glaucoma-associated optineurin mutants induce cell death selectively. Sci Rep. 2017; 7(1): 16855.

6. Shim MS, Kim KY, Noh M, Ko JY, Ahn S, An MA, et al. Optineurin E50K triggers BDNF deficiencymediated mitochondrial dysfunction in retinal photoreceptor cell line. Biochem Biophs Res Commun. 2018; 503(4): 2690-97. 
7. VanderWall KB, Huang KC, Pan Y, Lavekar SS, Fligor CM, Allsop AR, et al. Retinal Ganglion Cells With a Glaucoma OPTN(E50K) Mutation Exhibit Neurodegenerative Phenotypes when Derived from ThreeDimensional Retinal Organoids. Stem Cell Rep. 2020; 15(1): 52-66.

8. Mancino R, Martucci A, Cesareo M, Giannini C, Corasaniti MT, Bagetta G, et al. Glaucoma and Alzheimer Disease: One Age-Related Neurodegenerative Disease of the Brain. Curr Neuropharmacol. 2018; 16(7): 971-7.

9. Osawa T, Mizuno Y, Fujita Y, Takatama M, Nakazato Y, Okamoto K. Optineurin in neurodegenerative diseases. Neuropathology. 2011; 31(6): 569-74.

10. Shao Z, Wu J, Du G, Song H, Li SH, He S, et al. Young bone marrow Sca-1 cells protect aged retina from ischaemia-reperfusion injury through activation of FGF2. J Cell Mol Med. 2018. 22(12): 61766189.

11. Subramanian A, Tamayo P, Mootha VK, Mukherjee S, Ebert BL, Gillette MA, et al. Gene set enrichment analysis: a knowledge-based approach for interpreting genome-wide expression profiles. Proc Natl Acad Sci U S A. 2005; 102(43): 15545-50.

12. Huang DW, Sherman BT, Lempicki RA. Systematic and integrative analysis of large gene lists using DAVID bioinformatics resources. Nat Protoc. 2009; 4(1): 44-57.

13. Kramer A, Green J, Pollard JJ, Tugendreich S. Causal analysis approaches in Ingenuity Pathway Analysis. Bioinformatics (Oxford, England), 2014; 30(4): 523-30.

14. Hou Y, Dan X, Babbar M, Wei Y, Hasselbalch SG, Croteau DL, et al. Ageing as a risk factor for neurodegenerative disease. Nat Rev Neurol. 2019; 15(10): 565-81.

15. Jonas JB, Aung T, Bourne RR, Bron AM, Ritch R, Panda-Jonas S. Glaucoma. Lancet (London, England). 2017; 390(10108): 2183-93.

16. Thanos S, Bohm MR, Meyer ZHM, Prokosch-Willing V, Hennig M, Bauer D, et al. Role of crystallins in ocular neuroprotection and axonal regeneration. Prog Retin Eye Res. 2014; 42: 145-61.

17. Inagaki S, Kawase K, Funato M, Seki J, Kawase C, Ohuchi K, et al. Effect of Timolol on Optineurin Aggregation in Transformed Induced Pluripotent Stem Cells Derived From Patient With Familial Glaucoma. Invest Ophthalmol Vis Sci. 2018; 59(6): 2293-2304.

18. Mossmann D, Vogtle FN, Taskin AA, Teixeira PF, Ring J, Burkhart JM, et al. Amyloid- $\beta$ peptide induces mitochondrial dysfunction by inhibition of preprotein maturation. Cell Metab. 2014; 20(4): 662-9.

19. Cunnane SC, Trushina E, Morland C, Prigione A, Casadesus G, Andrews ZB, et al. Brain energy rescue: an emerging therapeutic concept for neurodegenerative disorders of ageing. Nat Rev Drug Discov.2020; 19(9): 609-33.

20. Williams PA, Harder JM, Foxworth NE, Cochran KE, Philip VM, Porciatti V, et al. Vitamin B modulates mitochondrial vulnerability and prevents glaucoma in aged mice. Science (New York, N.Y.). 2017; 355(6326): 756-60.

21. Munemasa Y, Kitaoka Y. Autophagy in axonal degeneration in glaucomatous optic neuropathy. Prog Retin Eye Res. 2015; 47: 1-18. 
22. Verkhratsky A, Parpura V, Pekna M, Pekny M, Sofroniew M. Glia in the pathogenesis of neurodegenerative diseases. Biochem Soc Trans. 2014; 42(5): 1291-301.

23. Brown GC, Neher JJ. Microglial phagocytosis of live neurons. Nat Rev Neurosci. 2014; 15(4): 209-16.

24. Wei $X$, Cho KS, Thee EF, Jager MJ, Chen DF. Neuroinflammation and microglia in glaucoma: time for a paradigm shift. J Neurosci Res. 2019; 97(1): 70-6.

25. Deng L, Pushpitha K, Joseph C, Gupta V, Rajput R, Chitranshi N, et al. Amyloid $\beta$ Induces Early Changes in the Ribosomal Machinery, Cytoskeletal Organization and Oxidative Phosphorylation in Retinal Photoreceptor Cells. Front Mol Neurosci. 2019; 12: 24.

26. Mirzaei M, Gupta VB, Chick JM, Greco TM, Wu Y, Chitranshi N, et al. Age-related neurodegenerative disease associated pathways identified in retinal and vitreous proteome from human glaucoma eyes. Sci Rep. 2017; 7(1): 12685.

27. Mirzaei M, Pushpitha K, Deng L, Chitranshi N, Gupta V, Rajput R, et al. Upregulation of Proteolytic Pathways and Altered Protein Biosynthesis Underlie Retinal Pathology in a Mouse Model of Alzheimer's Disease. Mol Neurobiol. 2019; 56(9): 6017-34.

28. Tezel, G. Oxidative stress in glaucomatous neurodegeneration: mechanisms and consequences. Prog Retin Eye Res, 2006; 25(5): 490-513.

29. Stroud DA, Surgenor EE, Formosa LE, Reljic B, Frazier AE, Dibley MG, et al. Accessory subunits are integral for assembly and function of human mitochondrial complex I. Nature. 2016; 538(7623): 1236.

30. Wu M, Gu J, Guo R, Huang Y, Yang M. Structure of Mammalian Respiratory Supercomplex I1III2IV1. Cell. 2016; 167(6): 1598-609.

31. Chalasani ML, Radha V, Gupta V, Agarwal N, Balasubramanian D, Swarup G. A glaucoma-associated mutant of optineurin selectively induces death of retinal ganglion cells which is inhibited by antioxidants. Invest Ophthalmol Vis Sci. 2007; 48(4): 1607-14.

32. Osborne NN, Nunez-Alvarez C, Joglar B, Del OS. Glaucoma: Focus on mitochondria in relation to pathogenesis and neuroprotection. Eur J Pharmacol. 2016; 787: 127-33.

33. SZA S, D Z, T H, L Y. Role of the AMPK pathway in promoting autophagic flux via modulating mitochondrial dynamics in neurodegenerative diseases: Insight into prion diseases. Ageing Res Rev. 2017; 40: 51-63.

34. DG H, FA R, SA H. AMPK: a nutrient and energy sensor that maintains energy homeostasis. Nature Reviews. Molecular cell biology. 2012; 13(4): 251-62.

35. L Y, Y C, SA T. Autophagy pathway: Cellular and molecular mechanisms. Autophagy. 2018; 14(2): 207-15.

36. G K, B L. Autophagic cell death: the story of a misnomer. Nature reviews. Molecular cell biology. 2008. 9(12): 1004-10.

37. Wong YC, Ysselstein D, Krainc D. Mitochondria-lysosome contacts regulate mitochondrial fission via RAB7 GTP hydrolysis. Nature. 2018; 554(7692): 382-6. 
38. Audano M, Schneider A, Mitro N. Mitochondria, lysosomes, and dysfunction: their meaning in neurodegeneration. J Neurochem. 2018; 147(3): 291-309.

39. Abu-Remaileh M, Wyant GA, Kim C, Laqtom NN, Abbasi M, Chan SH, et al. Lysosomal metabolomics reveals V-ATPase- and mTOR-dependent regulation of amino acid efflux from lysosomes. Science. 2017; 358(6364): 807-13.

40. Mauvezin C, Nagy P, Juhasz G, Neufeld TP. Autophagosome-lysosome fusion is independent of VATPase-mediated acidification. Nat Commun. 2015; 6: 7007.

41. Mangieri LR, Mader BJ, Thomas CE, Taylor CA, Luker AM, Tse TE, et al. ATP6V0C knockdown in neuroblastoma cells alters autophagy-lysosome pathway function and metabolism of proteins that accumulate in neurodegenerative disease. PLoS One. 2014; 9(4): e93257.

42. Meier S, Bell M, Lyons DN, Rodriguez-Rivera J, Ingram A, Fontaine SN, et al. Pathological Tau Promotes Neuronal Damage by Impairing Ribosomal Function and Decreasing Protein Synthesis. J Neurosci. 2016; 36(3): 1001-7.

43. Ding Q, Markesbery WR, Chen Q, Li F, Keller JN. Ribosome dysfunction is an early event in Alzheimer's disease. J Neurosci, 2005; 25(40): 9171-5.

44. London A, Benhar I, Schwartz M. The retina as a window to the brain-from eye research to CNS disorders. Nat Rev Neurol. 2013. 9(1): 44-53.

45. Lee CS, Larson EB, Gibbons LE, Lee AY, McCurry SM, Bowen JD, et al. Associations between recent and established ophthalmic conditions and risk of Alzheimer's disease. Alzheimers Dement. 2019; 15(1): 34-41.

46. Chiasseu M, Alarcon-Martinez L, Belforte N, Quintero H, Dotigny F, Destroismaisons L, et al. Tau accumulation in the retina promotes early neuronal dysfunction and precedes brain pathology in a mouse model of Alzheimer's disease. Mol Neurodegener, 2017; 12(1): 58.

47. Gupta VB, Chitranshi N, den Haan J, Mirzaei M, You Y, Lim JK, et al. Retinal changes in Alzheimer's disease- integrated prospects of imaging, functional and molecular advances. Prog Retin Eye Res, 2020: 100899.

48. Hart NJ, Koronyo Y, Black KL, Koronyo-Hamaoui M. Ocular indicators of Alzheimer's: exploring disease in the retina. Acta Neuropathol, 2016. 132(6): 767-787.

49. Ramirez Al, de Hoz R, Salobrar-Garcia E, Salazar JJ, Rojas B, Ajoy D, et al. The Role of Microglia in Retinal Neurodegeneration: Alzheimer's Disease, Parkinson, and Glaucoma. Front Aging Neurosci. 2017; 9: 214.

50. McKinnon SJ, Lehman DM, Kerrigan-Baumrind LA, Merges CA, Pease ME, Kerrigan DF, et al. Caspase activation and amyloid precursor protein cleavage in rat ocular hypertension. Invest Ophthalmol Vis Sci. 2002; 43(4): 1077-87.

51. Koronyo-Hamaoui M, Koronyo Y, Ljubimov AV, Miller CA, Ko MK, Black KL, et al. Identification of amyloid plaques in retinas from Alzheimer's patients and noninvasive in vivo optical imaging of retinal plaques in a mouse model. Neuroimage. 2011; 54 Suppl 1: S204- 17. 
52. Lee MJ, Lee JH, Rubinsztein DC. Tau degradation: the ubiquitin-proteasome system versus the autophagy-lysosome system. Prog Neurobiol. 2013; 105: 49-59.

53. Zhang $Y$, Chen $X$, Zhao Y, Ponnusamy M, Liu Y. The role of ubiquitin proteasomal system and autophagy-lysosome pathway in Alzheimer's disease. Rev Neurosci. 2017; 28(8): 861-8.

54. Luo R, Su LY, Li G, Yang J, Liu Q, Yang LX, et al. Activation of PPARA-mediated autophagy reduces Alzheimer disease-like pathology and cognitive decline in a murine model. Autophagy. 2020, 16(1): 52-69.

55. Feng W, Zhang Y, Wang Z, Xu H, Wu T, Marshall C, et al. Microglia prevent beta-amyloid plaque formation in the early stage of an Alzheimer's disease mouse model with suppression of glymphatic clearance. Alzheimers Res Ther. 2020; 12(1): 125.

56. Iqbal J, Zhang K, Jin N, Zhao Y, Liu X, Liu Q, et al. Alzheimer's Disease Is Responsible for Progressive Age-Dependent Differential Expression of Various Protein Cascades in Retina of Mice. ACS Chem Neurosci. 2019; 10(5): 2418-2433.

57. Fernandez-Nogales M, Santos-Galindo M, Hernandez IH, Cabrera JR, Lucas JJ. Faulty splicing and cytoskeleton abnormalities in Huntington's disease. Brain Pathol. 2016. 26(6): 772-8.

58. Hsieh, Y.C., et al., Tau-Mediated Disruption of the Spliceosome Triggers Cryptic RNA Splicing and Neurodegeneration in Alzheimer's Disease. Cell Rep. 2019; 29(2): 301-16.

59. Raj B, Blencowe BJ. Alternative Splicing in the Mammalian Nervous System: Recent Insights into Mechanisms and Functional Roles. Neuron. 2015; 87(1): 14-27.

60. Wax MB, Tezel G, Kawase K, Kitazawa Y. Serum autoantibodies to heat shock proteins in glaucoma patients from Japan and the United States. Ophthalmology. 2001. 108(2): 296-302.

61. Thanos S, Bohm MR, Meyer ZHM, Prokosch-Willing V, Hennig M, Bauer D, et al. Role of crystallins in ocular neuroprotection and axonal regeneration. Prog Retin Eye Res. 2014. 42: 145-61.

62. Munemasa Y, Kwong JM, Caprioli J, Piri N. The role of alphaA- and alphaB-crystallins in the survival of retinal ganglion cells after optic nerve axotomy. Invest Ophthalmol Vis Sci. 2009. 50(8): 3869-75.

63. Piri N, Kwong JM, Caprioli J. Crystallins in retinal ganglion cell survival and regeneration. Mol Neurobiol. 2013; 48(3): 819-28.

64. Mirzaei M, Gupta VK, Chitranshi N, Deng L, Pushpitha K, Abbasi M, et al. Retinal proteomics of experimental glaucoma model reveal intraocular pressure-induced mediators of neurodegenerative changes. J Cell Biochem, 2020.

\section{Figures}


a

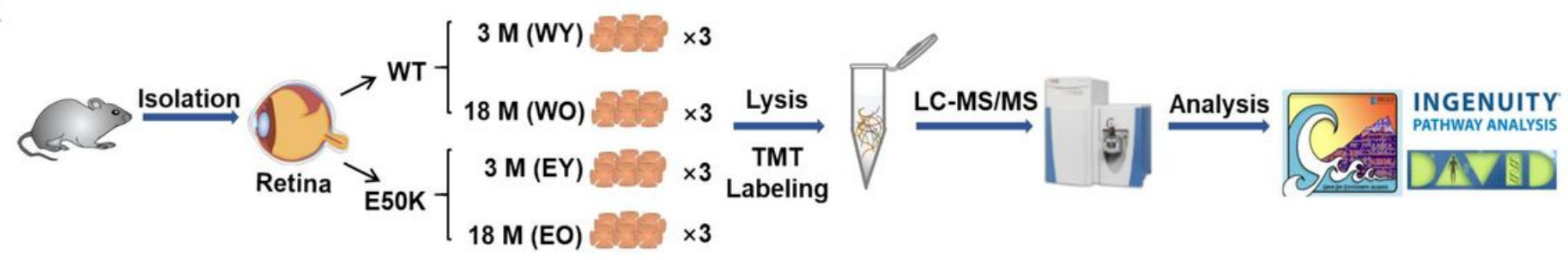

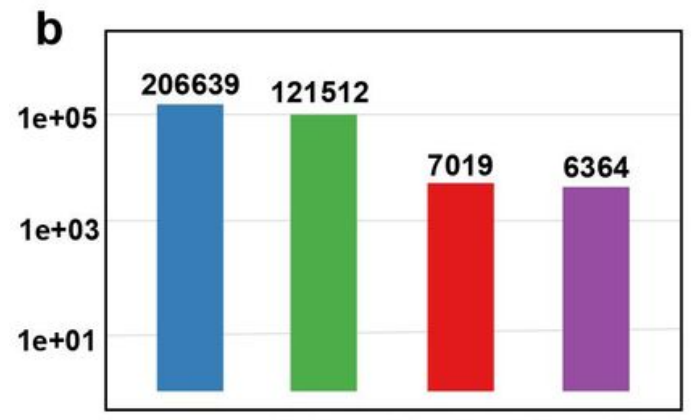

C

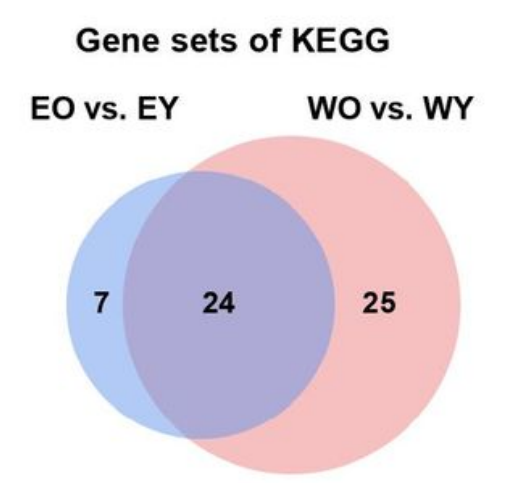

spectrum peptides proteins proteins

e

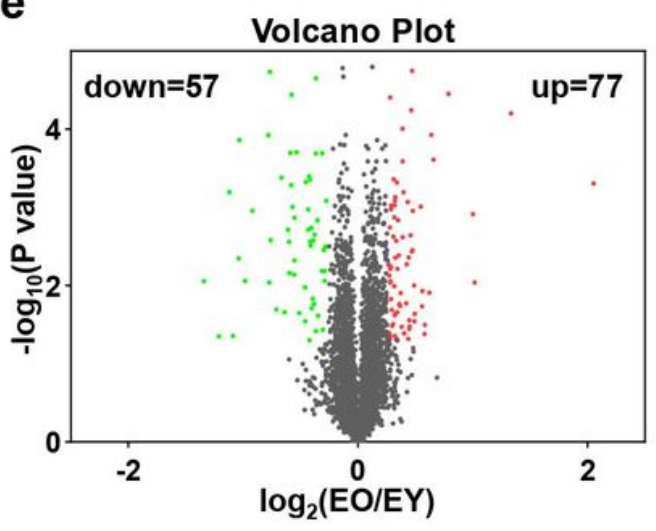

f

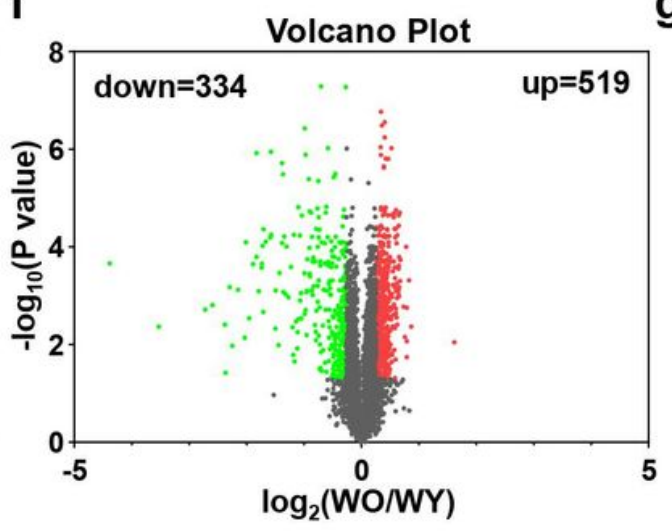

d

Gene sets of biological process

EO vs. EY WO vs. WY

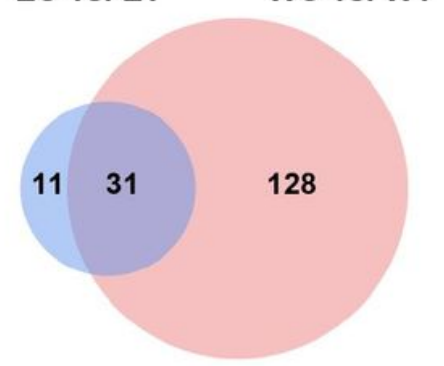

9 Differentially expressed proteins

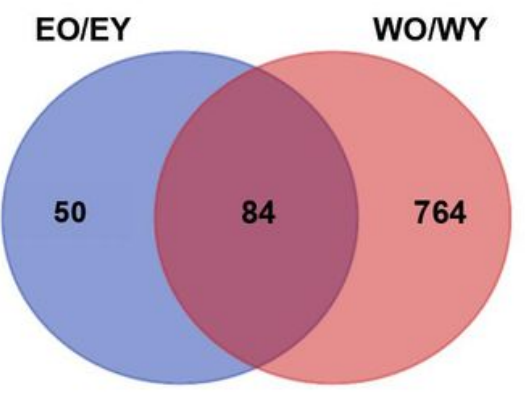

\section{Figure 1}

The role of the aging process in differential retinal protein expression. a Experimental workflow. Retinal proteins from both wildtype (WT) and OPTN E50K mutant (E5OK) mice, at 3- and 18-months were extracted ( 6 samples from 3 mice assigned into one biological replicate, $n=3$ replicates per group), then labelled with 10-plex tandem mass tag (TMT), and analyzed by liquid chromatography tandem mass spectrometry (LC-MS/MS). WY: Young WT; EY: Young E50K; WO: Old WT; EO: Old E50K. b Statistical information of mass spectrometry data results. A total of 7019 protein groups were identified, of which 6364 protein groups were quantified in all experimental sets. Quantifiable proteins were analyzed by gene set enrichment analysis (GESA), then compared with Kyoto Encyclopedia of Genes and Genomes (KEGG) pathways and biological processes (BP). Venn diagrams represent the significantly enriched (c) KEGG pathways and (d) BP in EO versus EY and WO versus WY. Volcano plots demonstrate the dual thresholds for differentially expressed proteins (DEPs) in (e) EO versus EY and (f) WO versus WY. Each dot represents a single quantified protein, and colored dots in upper and outer quadrants were considered $D E P s$, with green representing relative decrease and red increase in protein abundance $(F C \geq 1.2, P<0.05)$. (G) Venn diagram representing overlap of DEPs quantified in EO versus EY and WO versus WY groups. 
a

Molecular function
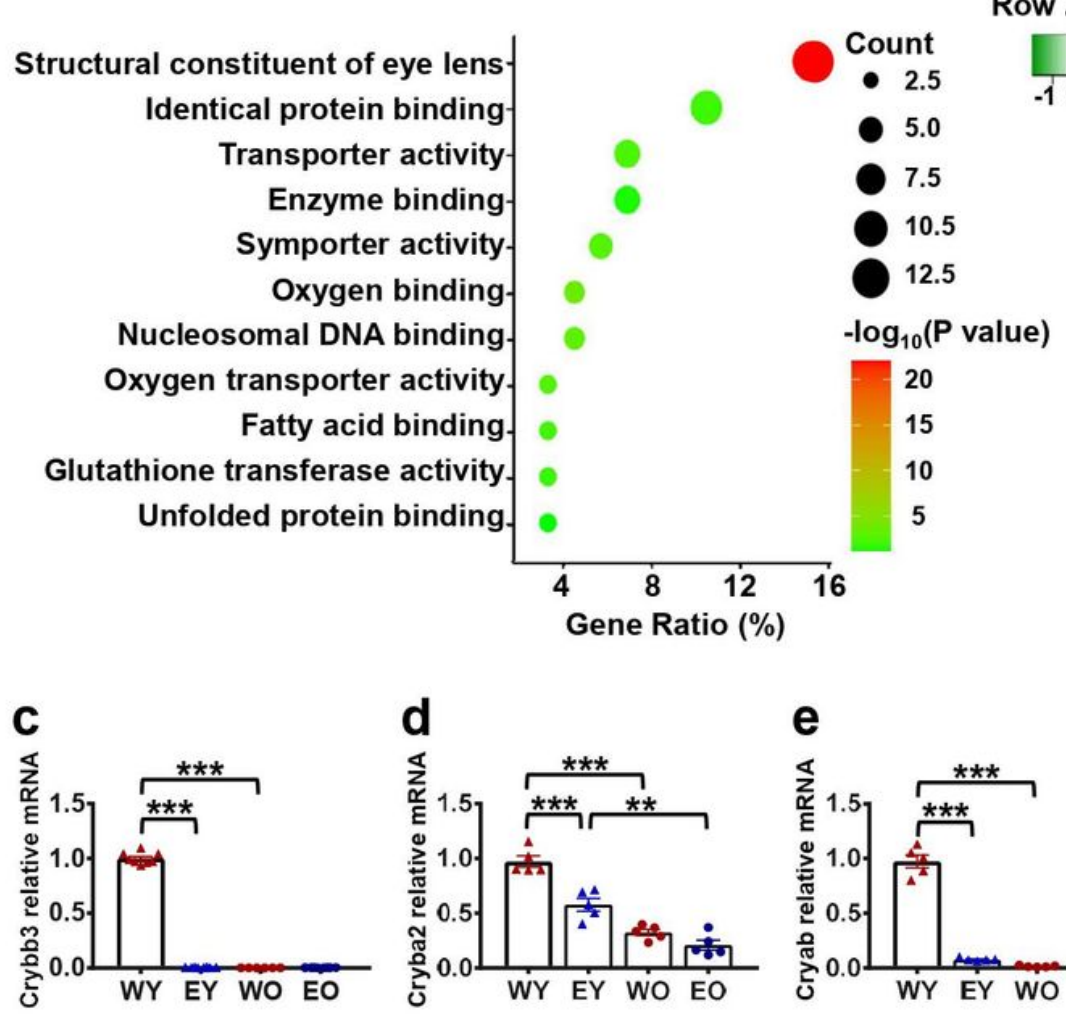

b

\section{Row Z-Score}

Crystallin family
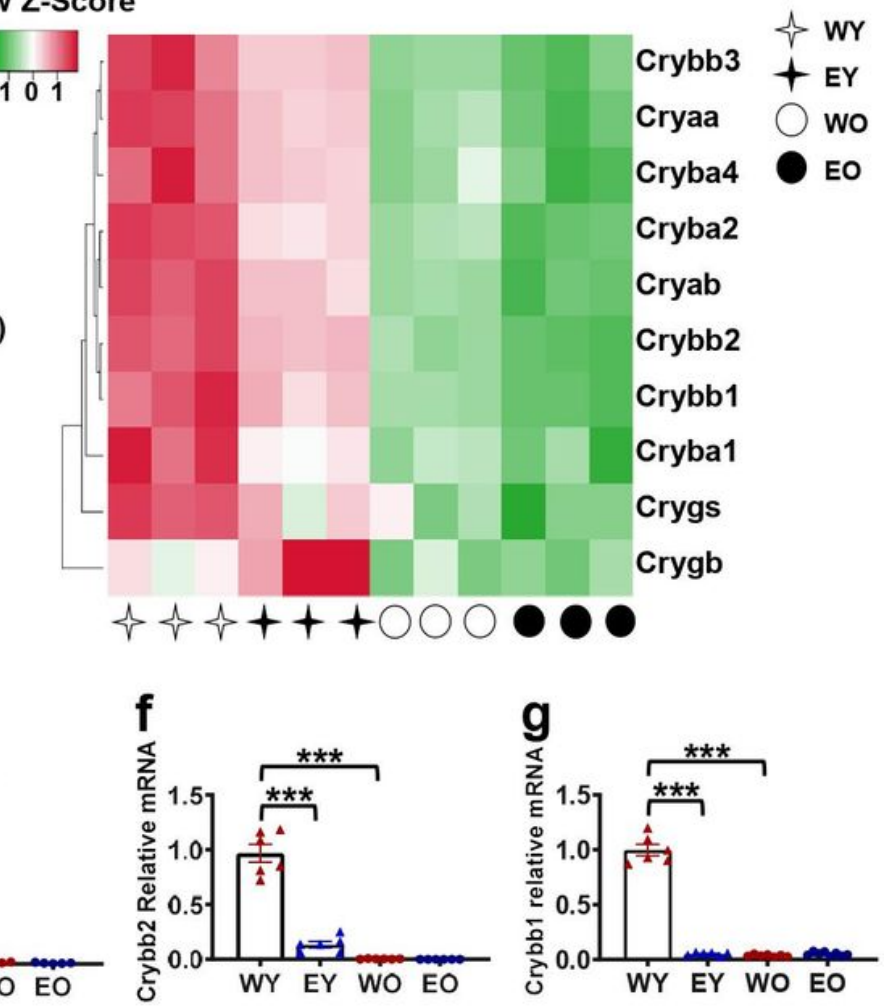

Figure 2

Crystallin family is the primary pathway associated with the aging process. a Bubble chart of the overlapped DEPs quantified in EO/EY and WO/WY by enriched Gene Ontology (GO) for molecular function in the Database for Annotation, Visualization, and Integrated Discovery (DAVID) database. Redder shades present in a bubble indicates greater statistical significance versus greener shades. Bubble size represents the counts of involved DEPs. b Heatmap showing relative protein expression for the crystallin family in four groups ( $\mathrm{n}=3$ per group). mRNA expressions of retinal (c) crystallin beta b3 (Crybb3), (d) Cryba2, (e) Cryab, (f) Crybb2, and (g) Crybb1 determined by real-time qPCR. Data shown as mean \pm SEM $\left(n=5\right.$, ONE WAY ANOVA followed by Tukey's post-hoc test, $\left.{ }^{*} P<0.05 ;{ }^{* \star P} P<0.01 ;{ }^{\star \star \star *} P<0.001\right)$. 
a

Gene sets of biological process

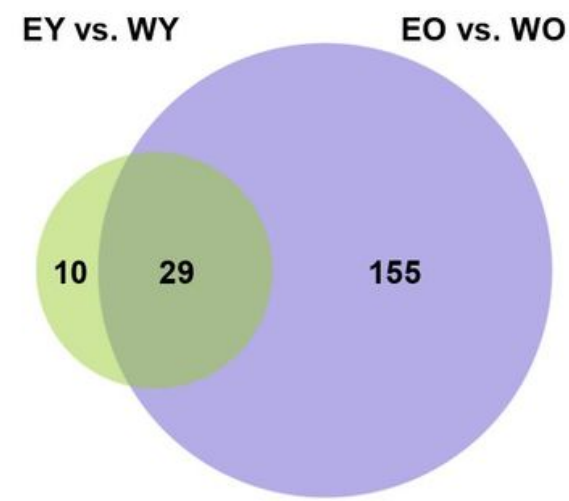

C

Transmembrane transport

Integrated protein rank

$0 \quad 2000 \quad 4000 \quad 6000 \quad 0 \quad 2000 \quad 4000 \quad 6000$

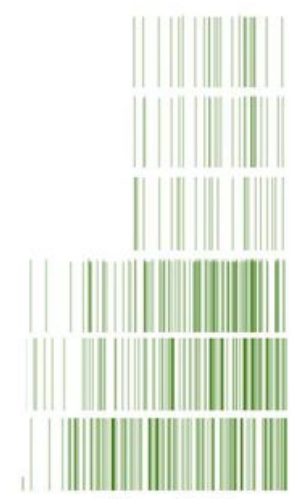

EY vs. WY

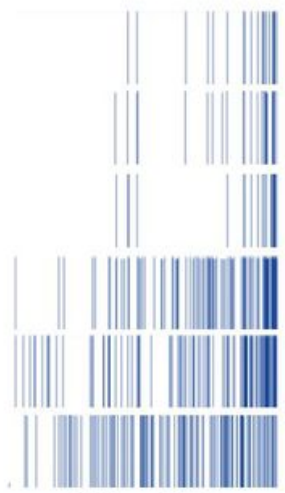

EO vs. WO Drug Anion b

mRNA splicing

Integrated protein rank

o 2000400060000200040006000

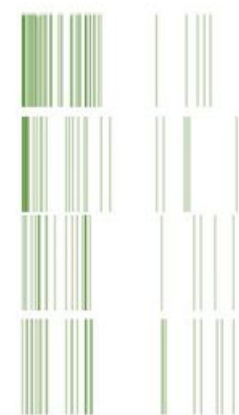

EY vs. WY

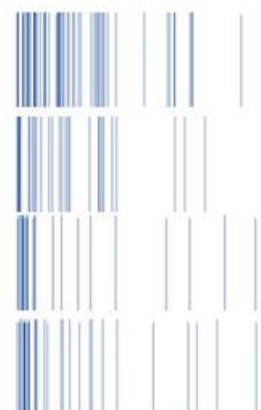

EO vs. Wo
Alternative mRNA splicing via spliceosome

mRNA splice site selection

Positive regulation of mRNA processing

Positive regulation of RNA splicing d

Enriched gene sets with opposite trend

L-amino acid transport

Amino acid transmembrane transport transmembrane transport transmembrane transport Organic acid transport Phospholipid biosynthesis
Protein localization to endoplasmic reticulum Establishing protein localization to endoplasmic reticulum

\begin{tabular}{lllllll}
\hline-3 & -2 & -1 & 0 & 1 & 2 & 3
\end{tabular}

\section{Figure 3}

The effect of OPTN E50K mutation on retinal protein biological processes. a Venn diagram representing significantly enriched biological processes in EY versus WY and EO versus WO by GSEA. Overlapping biological processes are categorized into 2 groups comprising (b) mRNA splicing and (c) transmembrane transport. Barcode plots represent the protein positions in the sorted integrated ranking. $d$ Eight overlapping biological processes showing opposite trends between EY versus WY and EO versus WO groups. 
a

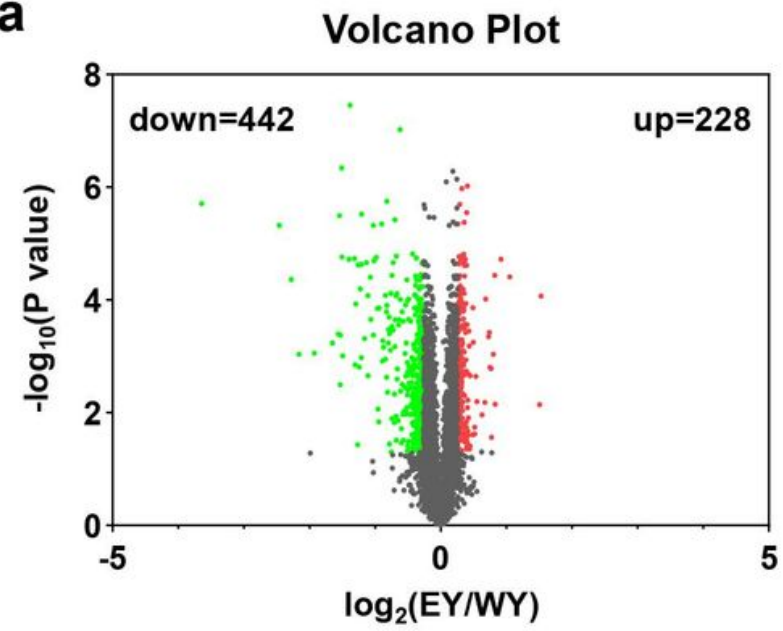

C

\section{Overlapped proteins}

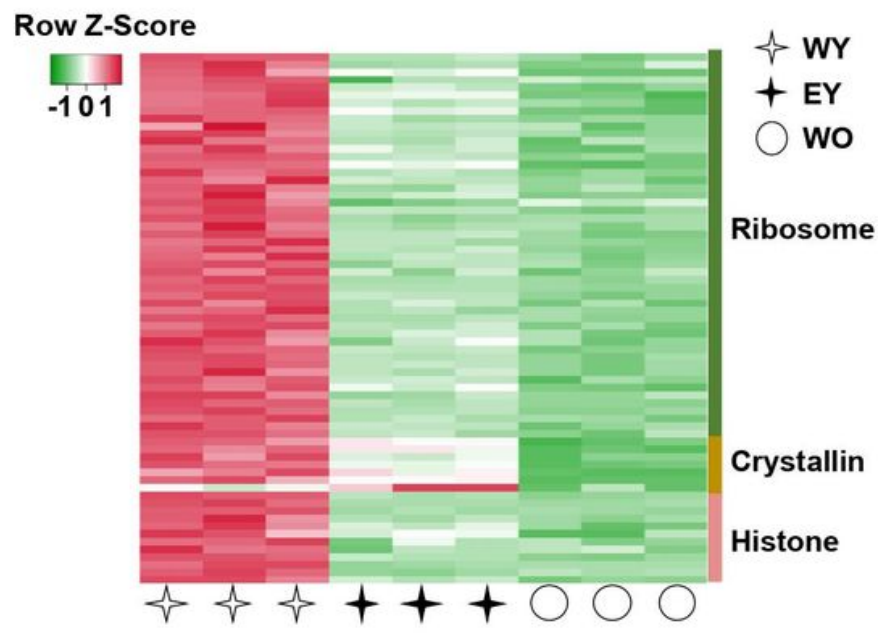

b
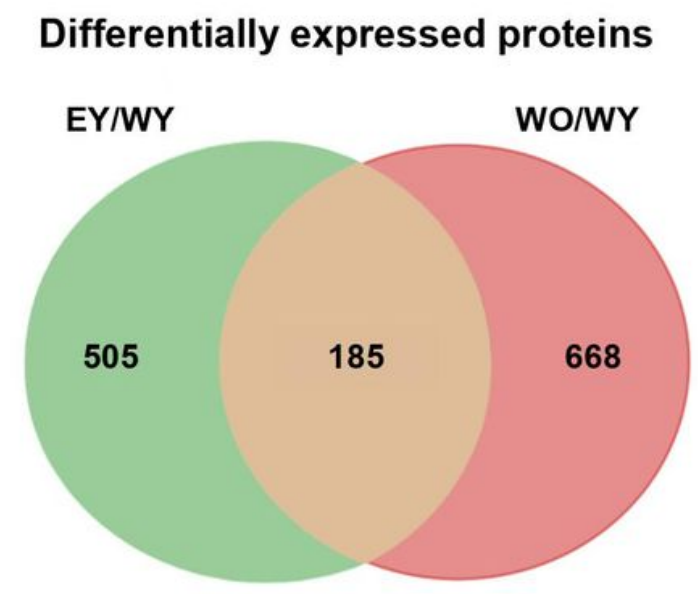

d

Canonical pathway

\section{Figure 4}

Young OPTN-E50K mutant mice have similar protein expression profiles as old wild-type mice. a Volcano plot demonstrates the dual thresholds for DEPs in EY compared to WY. Each dot represents a single quantified protein. Dots with color in upper and outer quadrants were considered DEPs, with green indicating relative decrease, and red increase in protein abundance $(\mathrm{FC} \geq 1.2$ and $\mathrm{P}<0.05)$. b Venn diagram represents the overlap of DEPs quantified in EY/WY WO/WY groups. c Heatmap depicting the relative expression of the overlapped proteins, comprising ribosomal, crystallin family, and histone proteins in three groups ( $n=3$ per group). $d$ Bubble chart indicating the top 6 canonical pathways enriched in IPA analysis based on EY and WO DEPs. Redder shades present in a bubble indicates greater statistical significance versus greener shades. Bubble size represents the counts of involved DEPs. 
a

Volcano Plot

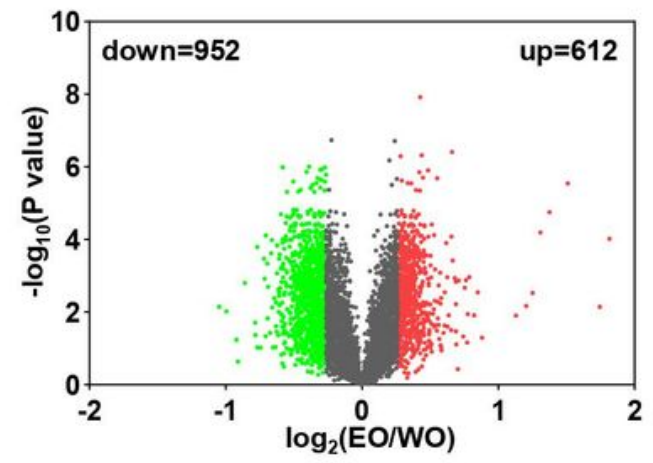

C

Diseases or functions annotation

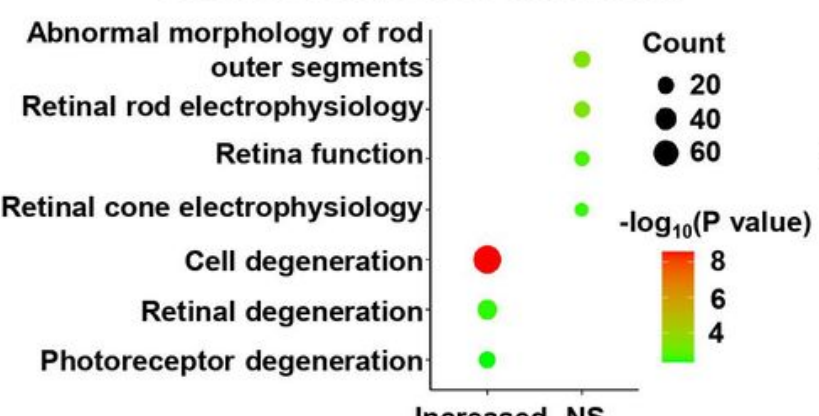

Increased NS b

Differentially expressed proteins

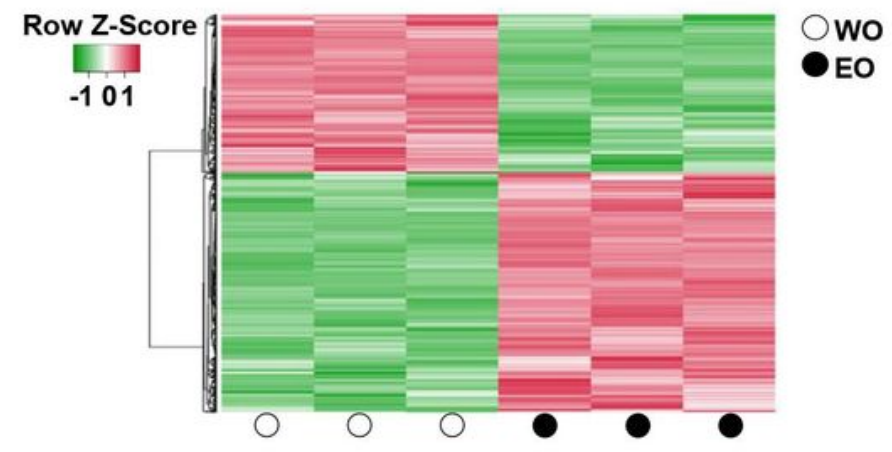

d

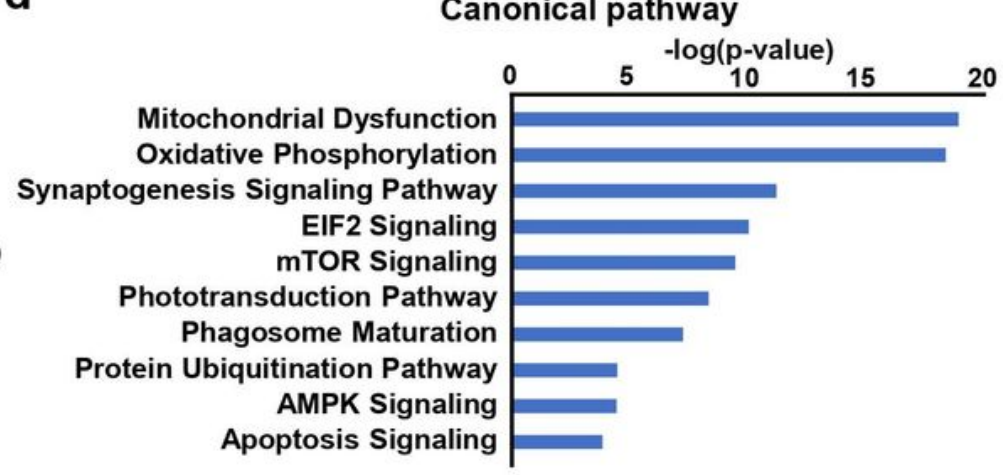

e

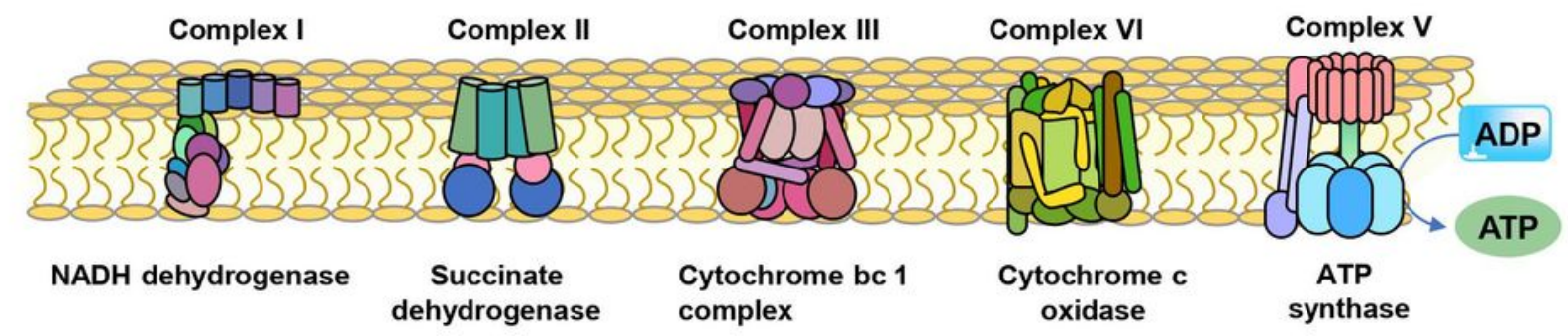

f

\begin{tabular}{|c|c|c|c|c|c|c|c|}
\hline \multicolumn{2}{|c|}{ Complex I } & Complex II & Complex III & Complex IV & Com & lex V & \multirow{11}{*}{$\begin{array}{l}\text { Down } \\
\text { Up }\end{array}$} \\
\hline Ndufa3 & Ndufa5 & Sdhe & Cyc1 & $\mathrm{mt}-\mathrm{Co} 2$ & Atp5j2 & Atp4b & \\
\hline Ndufa9 & Ndufa6 & & & mt-Co3 & Atp5k & Atp5a1 & \\
\hline Ndufa13 & Ndufa7 & & & Cox4i1 & Atp5pb & Atp5b & \\
\hline Ndufb3 & Ndufab1 & & & Cox4i2 & Atp6ap1 & Atp5c1 & \\
\hline Ndufb4 & Ndufs4 & & & Cox7a2 & Atp6v0a1 & Atp5d & \\
\hline Ndufb5 & Ndufs5 & & & Cox7c & Atp6v0c & Atp5j & \\
\hline Ndufb6 & Ndufs6 & & & Cox6c & Atp6v1c1 & Atp6v1e1 & \\
\hline Ndufb7 & Ndufv3 & & & Cox5a & Atp12a & Atp6v1f & \\
\hline Ndufb8 & & & & Cox17 & mt-Atp6 & & \\
\hline Ndufb11 & & & & & mt-Atp8 & & \\
\hline
\end{tabular}

Figure 5

Canonical pathways and proteins affected by E50K Mutation in old normal tension glaucoma (NTG) mice. a Volcano plot demonstrating the dual thresholds for DEPs in EO versus WO mice. Each dot represents a single quantified protein. Dots with color in the upper and outer quadrants were DEPS, with green indicating relative decrease, and red increase in abundance ( $F C \geq 1.2$ and $\mathrm{P}<0.05)$. b Hierarchical clustered heatmap depicting individual sample and gene expression differences between EO and WO 
( $\mathrm{n}=3$ per group). $\mathrm{c}$ Bubble chart depicting the top significant diseases and biological functions determined by IPA. Redder shades present in a bubble indicates greater statistical significance versus greener shades. Bubble size represents the counts of involved DEPs. d Horizontal bar chart depicting significantly enriched canonical pathways, based on P-values. e A schematic diagram representing the mitochondrial complex subunit in aged NTG condition. $f$ Mitochondrial complex subunit DEPs associated with up- or down-regulation under aged NTG conditions. Nduf: NADH-ubiquinone oxidoreductase, mt-ND: Mitochondrially-encoded NADH-ubiquinone oxidoreductase, Sdhc: Succinate dehydrogenase complex subunit C, Cyc1: Cytochrome c1, mt-Co: Mitochondrially-encoded cytochrome c oxidase, Cox: Cytochrome c oxidase, Atp: ATP synthase.
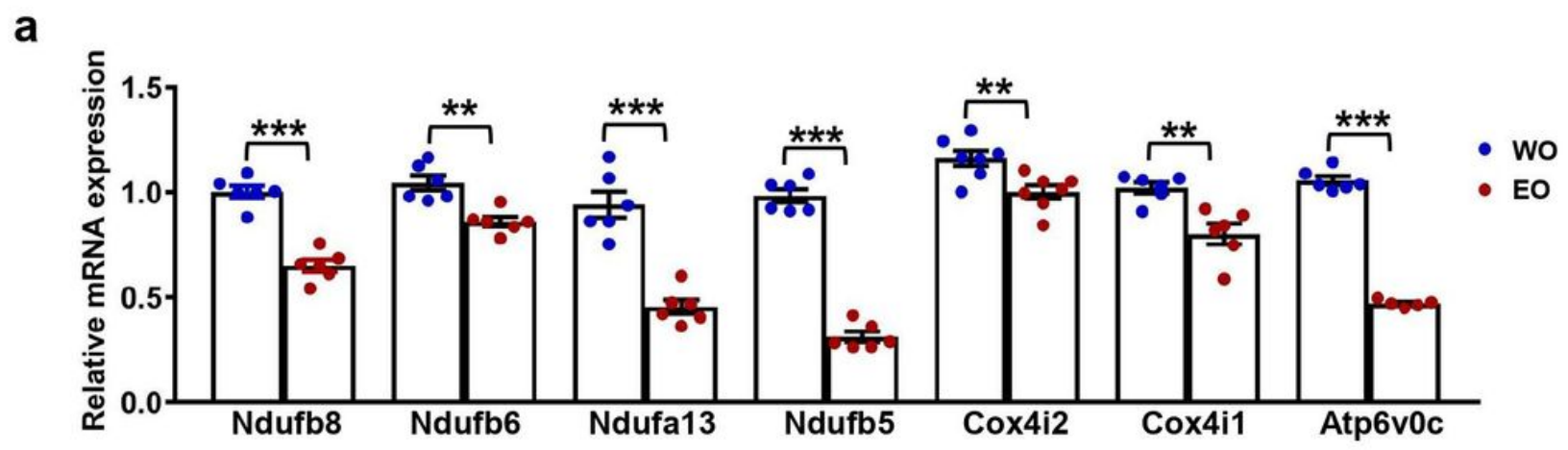

b

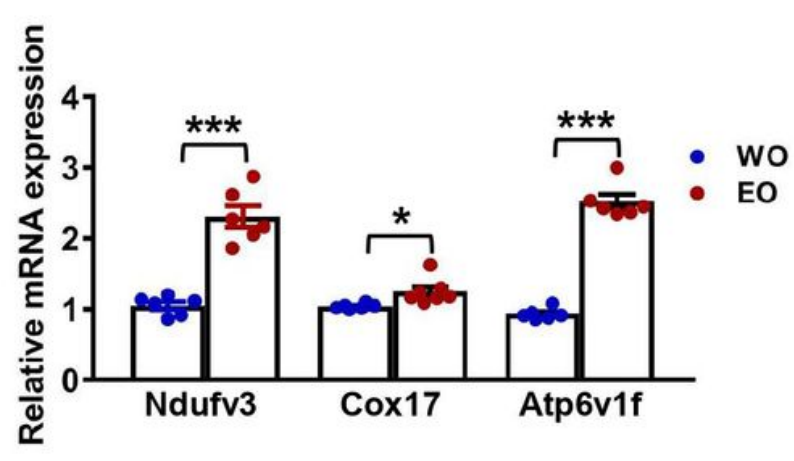

C

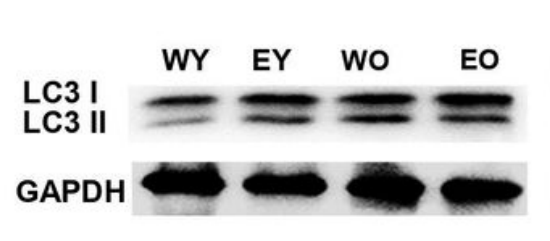

d

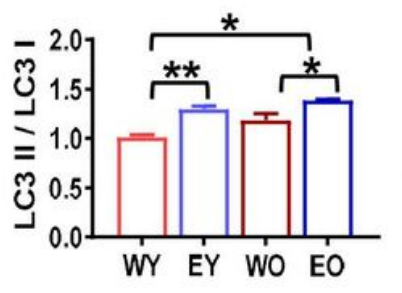

e

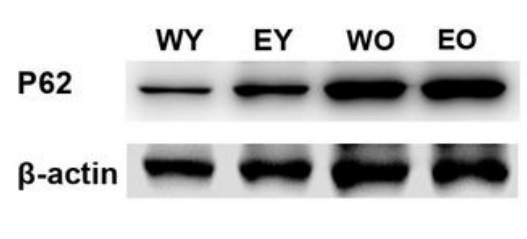

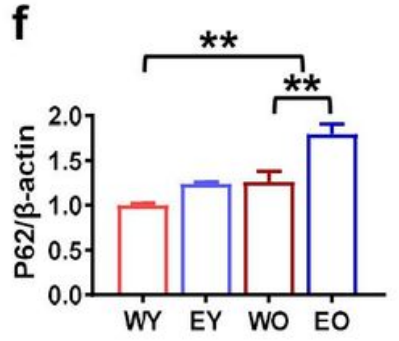

Figure 6

Quantitative validation of mitochondrial subunit and autophagy defects in old E50K mice. a-b Real-time qPCR validation of mRNA expression levels for selected mitochondrial subunit proteins. Bar charts depicted (a) downregulated of Ndufb8, Ndufb6, Ndufa13, Ndufb5, Cox4i2, Cox4i1 and Atp6v0c and (b) upregulated Ndufv3, Cox17 and Atp6v1 $f$ in EO compared to WO group. Data shown as mean \pm SEM ( $n=5$, Student's t-test, $\left.{ }^{*}<<0.05,{ }^{*} \mathrm{P}<0.01,{ }^{*} * \mathrm{P}<0.001\right)$. c-f Representative Western blot images and 
a

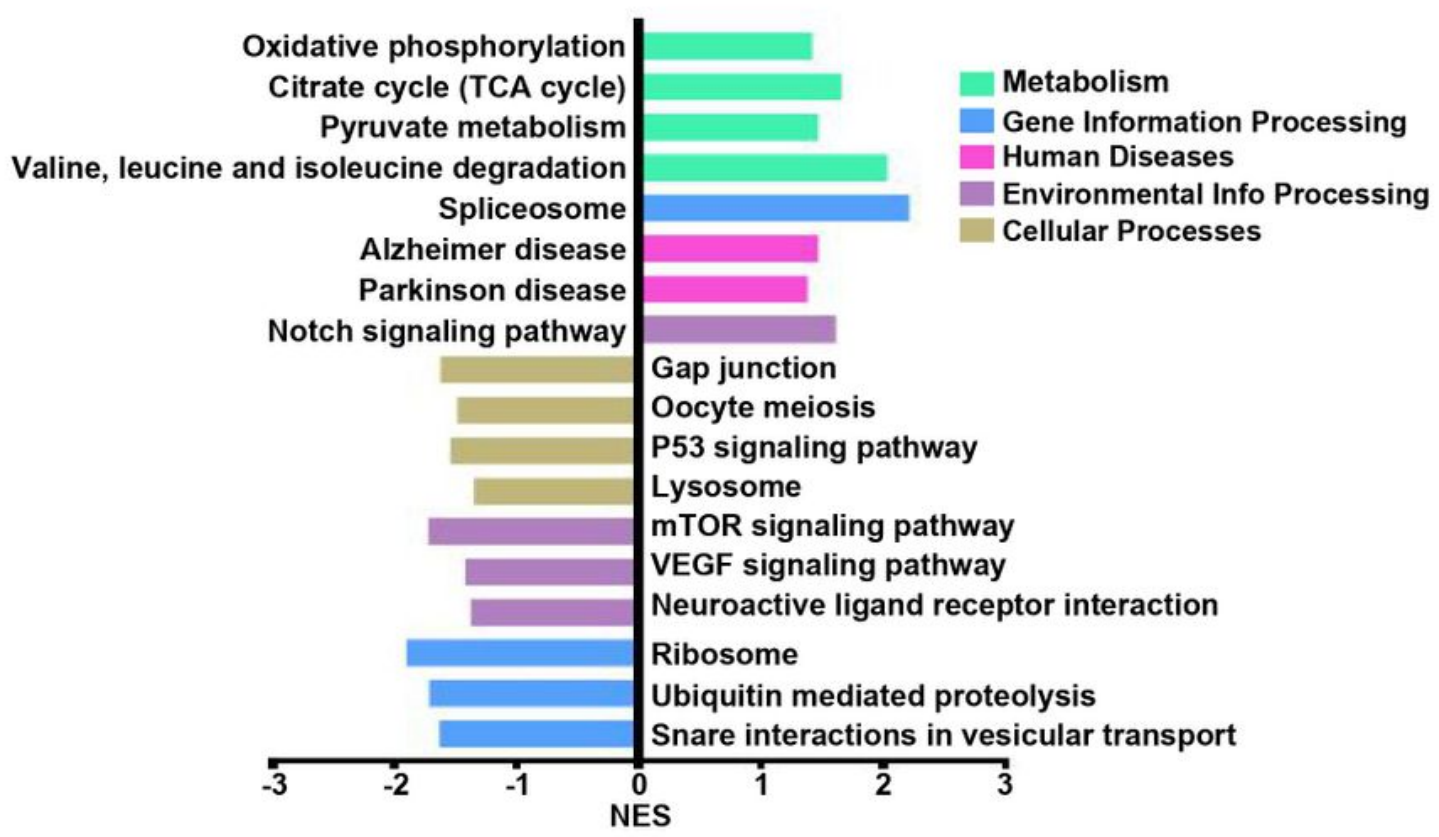

b

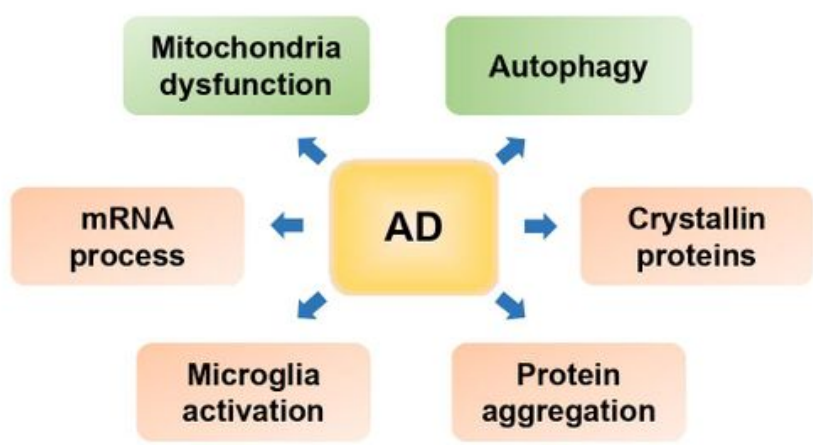

C

KEGG-Spliceosome

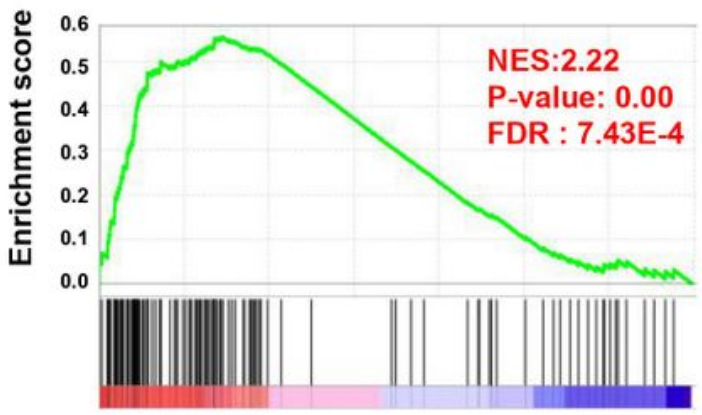

d

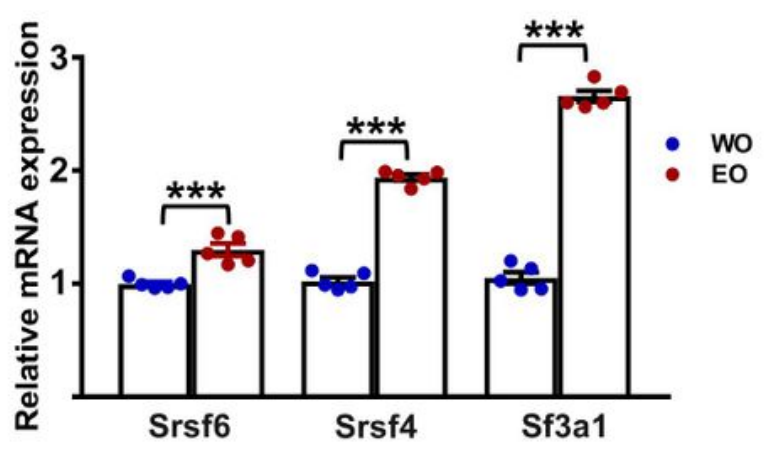

Figure 7

NTG shares similar biochemical enrichment in the spliceosome as with Alzheimer's disease. a Significantly enriched KEGG pathways in EO compared with other 3 groups by GSEA. b Reported diseaserelated biological changes commonly enriched in Alzheimer's disease (AD). c Enrichment plot of 
spliceosome pathway from GSEA. d Bar chart depicting mRNA expression of spliceosome proteins serine/arginine-rich splicing factor (Srsf) 6, Srsf4, and splicing factor 3 subunit 1 (Sf3a1) in EO compared to WO retina using real-time qPCR. Data shown as mean \pm SEM $\left(n=5\right.$, Student's t-test, $\left.{ }^{* \star *} P<0.001\right)$.

a
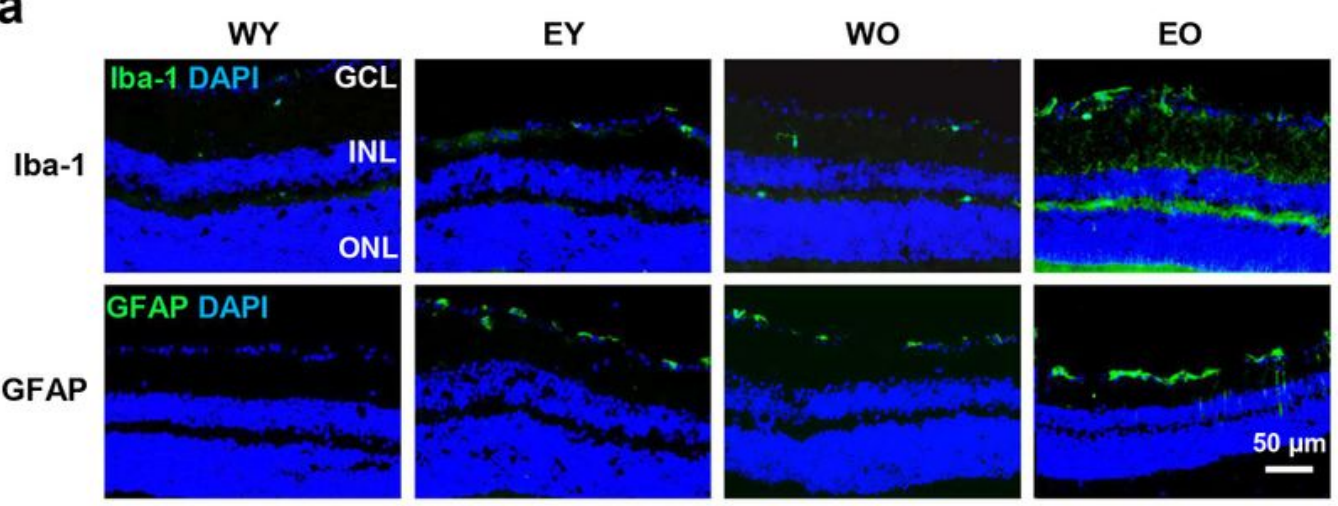

b
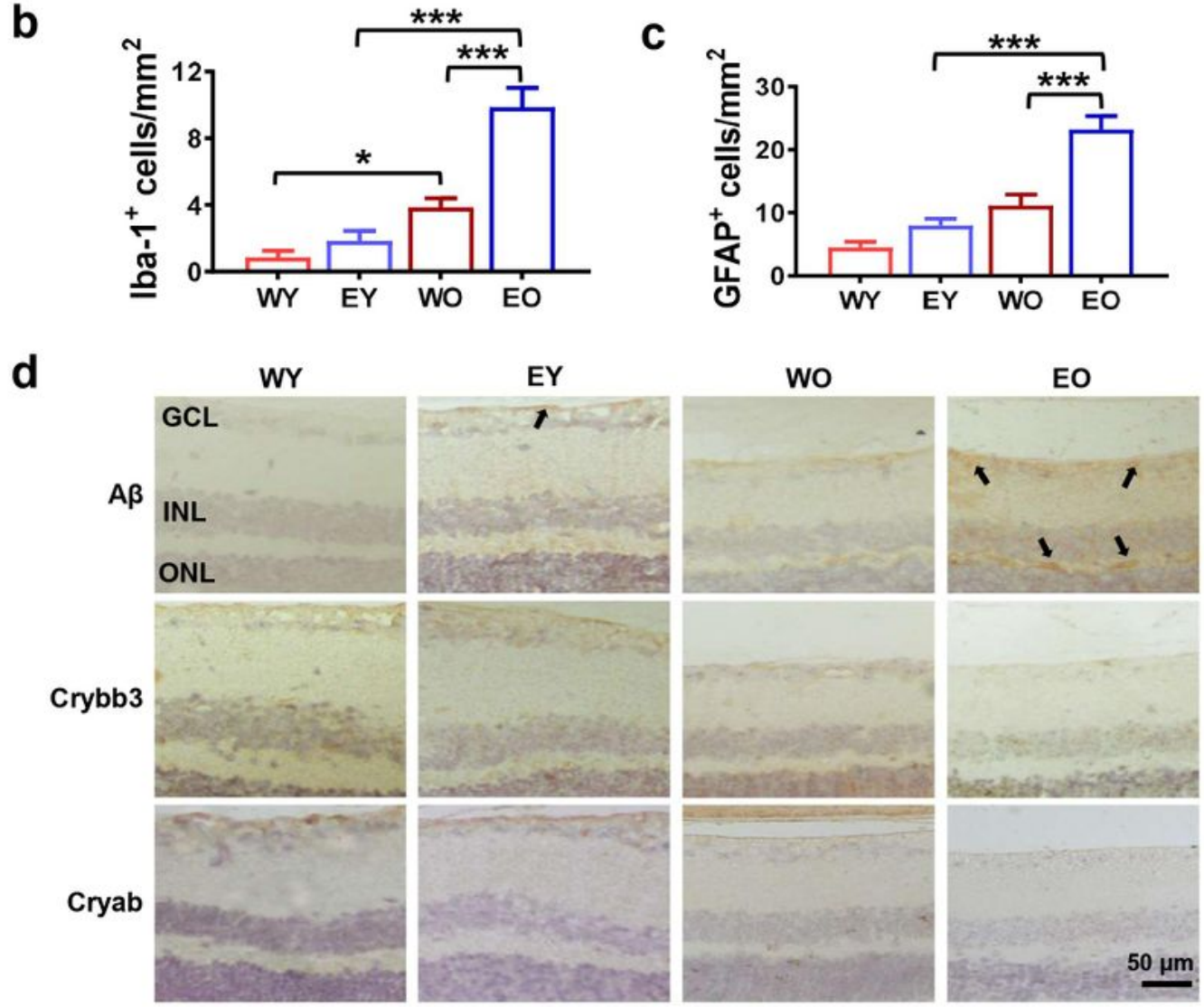

e
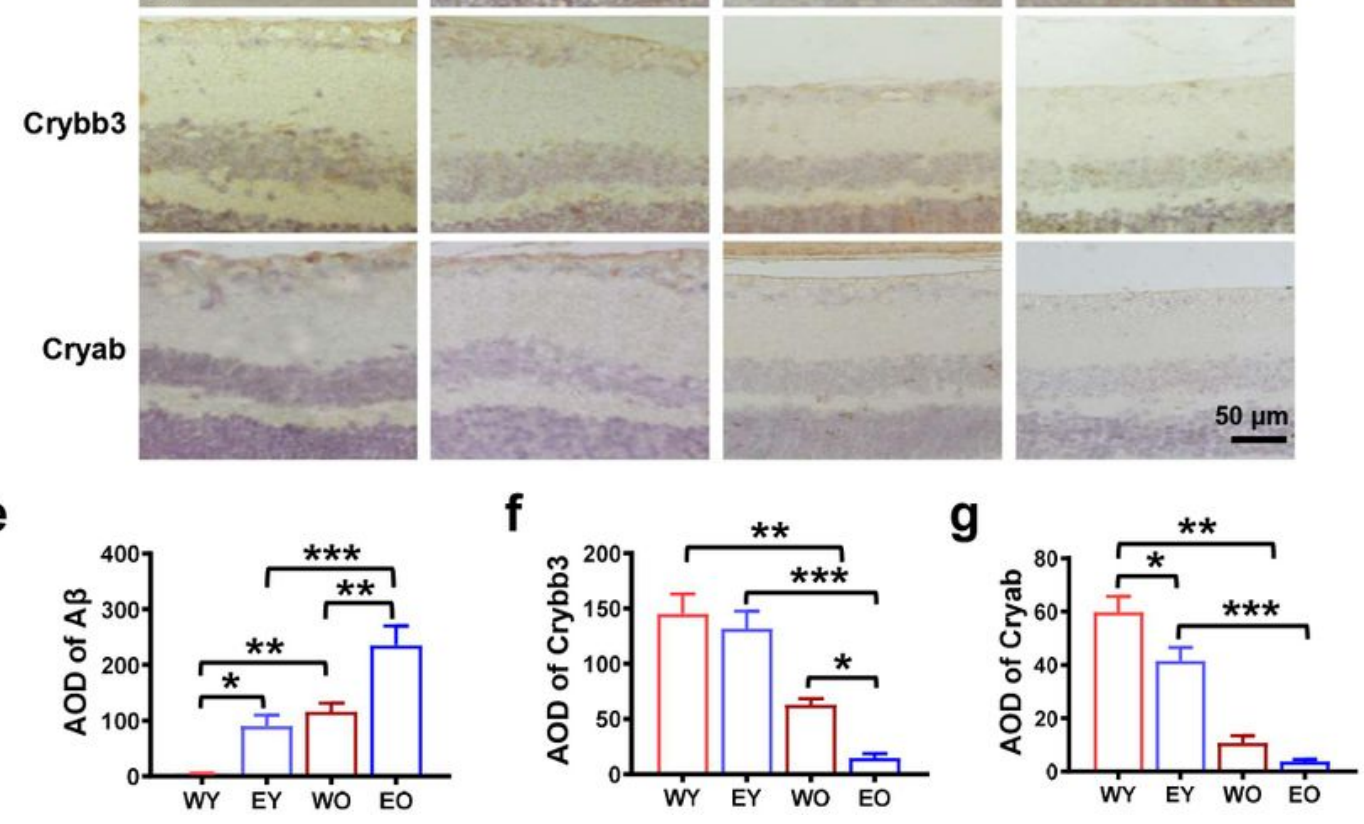

Figure 8

NTG shares the pathological phenotypes of glial cell activation and $\beta$-amyloid aggregation, associated with Alzheimer's disease. a Retinal sections were immuno-stained for ionized calcium binding adaptor 
molecule 1 (Iba-1) and glial fibrillary acidic protein (GFAP) in all 4 groups. Cell nuclei were visualized with DAPI. The number of (b) Iba-1+ cells and (c) GFAP+ cells in the ganglion cell layer (GCL) was significantly increased in EO mice compared to the other 3 groups. $d$ Immunostaining of $\beta$-amyloid (A $\beta), C r y b b 3$ and Cryab proteins in all 4 groups. $A \beta$ expression in $E Y$ and $E O$ in retinal sections was indicated by black arrows. Positive areas for (e) $A \beta$, (f) Crybb3 and (g) Cryab were detected for all 4 groups. Data shown as mean \pm SEM $\left(n=3\right.$, ONE WAY ANOVA followed by Tukey's post-hoc test, $\left.{ }^{*} P<0.05, * * P<0.01 ; * \star * P<0.001\right)$.

\section{Supplementary Files}

This is a list of supplementary files associated with this preprint. Click to download.

- GraphicAbstract.pdf

- Additionalfile1.docx

- Additionalfile2.docx

- Additionalfile3.docx

- SupplementalFigureS1.pdf

- SupplementalFigureS2.pdf

- SupplementalFigureS3.pdf

- SupplementalFigureS4.pdf

- SupplementalFigureS5.pdf

- Highlights.docx 